\title{
Substituent Effects on the Activation Parameter Changes for the Aminolysis in the Bimolecular Nucleophilic Reactions in Solution
}

\author{
Vladislav M. Vlasov
}

\begin{abstract}
N. N. Vorozhtsov Novosibirsk Institute of Organic Chemistry, Siberian Branch of the Russian Academy of Sciences, 630090 Novosibirsk, Lavrentjev Ave., 9, Russian Federation
\end{abstract}

\begin{abstract}
Variation of the activation parameters for the aminolysis in the $S_{N} 2$, acyl-transfer, $S_{N} A r$ and $A d_{N}$ reactions offers an additive mechanistic tool for the studies of these reactions in solution. This approach uses the substituent effects on the benzene and pyridine rings to the variation of the activation parameters, $\Delta X^{*}(X=H, S, G)$, in the above reactions in the frameworks of the Hammett - like equations in order to evaluate the resultant $\delta \Delta X^{\neq}$reaction constants. The single linear dependences of the internal enthalpy constants $\delta \Delta H^{\ddagger}$ int on the $\delta \Delta G^{\neq}$and the Hammett $\rho$ constants show that the substituent effects in the leaving and nonleaving groups and nucleophiles on the mechanistic features in aminolysis of bimolecular nucleophilic reactions are governed by the magnitude of $\delta \Delta H^{\ddagger}$ int when one of the steps of the process is the single rate-determining step.
\end{abstract}

Keywords: Aminolysis in solution, nucleophilic reactions, activation parameters, reaction mechanism.

\section{INTRODUCTION}

A significant part of reactions carried out by the pharmaceutical and agrochemical industries involve aminolysis in the bimolecular nucleophilic reactions (BNRs) in solution [1,2]. The aminolysis with amines and pyridines in the BNRs plays also an important role for both organic chemistry $[3,4]$ and biochemistry [5]. These include $S_{N} 2$, acyl-transfer, $S_{N} A r$ and $A d_{N}$ reactions, etc [3-6]. The nature of the reactants or solvents influences both the kinetics and mechanisms of these reactions $[7,8]$. Various experimental kinetic and theoretical studies have therefore been devoted to obtain a better understanding of the mechanisms of the aminolysis in the nucleophilic reactions of the substitution and addition [5-19]. Among traditional experimental methods, kinetic isotope effects [8,20-22] and linear free energy relationships [23-26] have most frequently been used to study mechanisms of BNRs, in particular the nature of transition states (TSs) [27-29].

The activation parameters are widely used for characterizing the TS structures for BNRs $[8,23-27,30]$. Some generalizations of the changes in the enthalpy of activation $\left(\Delta H^{\ddagger}\right)$, the entropy of activation $\left(\Delta S^{\neq}\right)$, and the activation free energy $\left(\Delta G^{\neq}\right)$for these reactions were made $[23,30]$. The influence of the variations of substituents in substrates and amines on activation parameters in $S_{N} 2$, acyl-transfer, $S_{N} A r$ and $A d_{N}$ reactions was demonstrated [30-36]. The effects of substituents on the aromatic ring were used in order to

${ }^{*}$ Address correspondence to this author at the N. N. Vorozhtsov Novosibirsk Institute of Organic Chemistry, Siberian Branch of the Russian Academy of Sciences, 630090 Novosibirsk, Lavrentjev Ave., 9, Russian Federation; Tel: +7(383)330-88-33; Fax: +7(383)330-97-52;

E-mail: vmvlasov@nioch.nsc.ru evaluate the contribution of changes of the activation parameters in these reactions in the frameworks of the Hammett - like equation (1) $[37,38]$.

$\Delta X^{\neq}=\delta \Delta X^{\neq} \sigma+\Delta X_{0}^{\neq}(X=H, S, G)$

In this equation, $\sigma$ is the Hammett constant of the substituent, the slope $\left(\delta \Delta X^{\ddagger}\right)$ is the selectivity of its influence on the activation parameters $\Delta X^{*}(X=H, S$, $G)$, and the term $\Delta X_{0}^{\neq}$is the activation parameters for the unsubstituted compound. Therefore, the reaction constant $\delta \Delta X^{\neq}$is analogous to the Hammett one $\rho$.

Recent analysis of the reaction constants, $\delta \Delta X^{\ddagger}(X$ $=H, S, G)$, has revealed a number of $\delta \Delta H^{\neq}$versus $\delta \Delta S^{\neq}$compensation equations for typical BNRs for which a substituent is varied on the leaving and nonleaving groups, as well as on the charged and neutral nucleophiles [31]. In addition, the variations of the reaction constants $\delta \Delta X^{*}$ [eqn (1)] were discussed in the context of estimating the changes in the internal and external reaction constants, $\delta \Delta X_{\text {int }}^{\neq}$and $\delta \Delta H_{\text {ext }}^{\neq}$, respectively [eqn (2)] [31].

$\delta \Delta X^{\neq}=\delta \Delta X_{\text {int }}^{\neq}+\delta \Delta X_{\text {ext }}^{\neq}$

In keeping with the Hepler solvation theory $[37,38]$, in this equation the reaction constants $\delta \Delta X^{\neq}$are divided into internal $\left(\delta \Delta X_{\text {int }}^{\neq}\right)$and external $\left(\delta \Delta X_{\text {ext }}^{*}\right)$ terms, which refer to the chemical reaction and the solvation process, respectively [31-36]. It was found that a single Hammett-like linear dependences between $\delta \Delta H^{*}$ int versus $\delta \Delta G^{\neq}$and $\delta \Delta H^{\neq}$int versus $\rho$ for the BNRs have been developed for which the $k_{\mathrm{c}}$ or $k_{1}$ steps were ratedetermining [eqn (3) and (4)] (Scheme 1) [31]. 
$\delta \Delta H_{\text {int }}^{\neq}=(-0.21 \pm 0.15)+(1.00 \pm 0.01) \delta \Delta G^{\neq}$

$r=0.996, s=1.29, n=82$

$\delta \Delta H_{\text {int }}^{ \pm}=(-0.3 \pm 0.2)-(6.0 \pm 0.1) \rho$

$r=0.994, s=1.7, n=82$

This scheme shows the main mechanisms of the aminolysis in the $S_{N} 2$ [6-8], acyl-transfer [5], $S_{N} A r$ [1416] and $\mathrm{Ad}_{N}$ reactions [19] proceeding both by concerted and stepwise pathways. It should be noted that the mechanism of aminolysis with neutral amines in $S_{N} 2[31,33-35,39]$, and $\mathrm{Ad}_{N}$ reactions [40-43] is concerted proceeding through a single TS with the $k_{\mathrm{c}}$ step as the rate-determining one (Scheme 1). At the same time the aminolysis in the acyl-transfer and $S_{N} A r$ reactions proceed by a different mechanisms. The aminolysis with neutral amines in the acyl-transfer reactions can follow both a concerted [44,45] and a stepwise mechanism [31,32,46-53], for which the nucleophilic attack with the $k_{\mathrm{c}}$ and $k_{1}$ (or $k_{2}$ ) steps are to be rate-determining, respectively. The nucleophilic substitution mechanism $\mathrm{S}_{N} \operatorname{Ar}$ (addition - elimination) in arenes with neutral amines depends on the reaction medium and substrate structure [14-17]. Normally, general base catalysis occurs in nonpolar aprotic solvents $[54,55]$. However, in dipolar aprotic solvents the general base catalysis is not observed [56-58]. Obviously, the formation of $\sigma$ complex (rate constant $k_{1}$ ) and its decomposition (rate constants $k_{2}$ or $k_{3}[=\mathrm{NH}]$ ) are presumed to be rate - determined [14-17,54-61]. If in a stepwise reaction an addition of amine to the substrate (rate constant $k_{1}$ in Scheme 1) or a decomposition of intermediate (rate constant $k_{2}$ ) is the single rate - determining step (RDS), it can be supposed that the effect of the substituents $R$ in the leaving group of compounds $3-14$, the nonleaving group of compounds $20-23,25,27,30-33$ and nucleophiles $\mathbf{3 6}-\mathbf{3 8}$ on the changes of the reaction constants $\delta \Delta X^{\neq}(X=H, S, G)$ can be similar in according to eqns (3) and (4) for all BNRs proceeding also by concerted mechanism (cf. ref. 62,63). Therefore, we considered it of interest to address these questions in the present work. Furthermore, an additional focus of our interest in the properties of the RDS concerned the single RDS for the aminolysis in the multistep BNRs.

Herewith, we report on the effects of the substituents in the leaving and nonleaving groups, as well as in the nucleophiles on the variation in the $\delta \Delta X^{\neq}$ $(X=H, S, G)$ values and on the mechanisms for the typical BNRs with neutral amines and pyridines in protic and aprotic media (Scheme 1). Our second aim in this work was to extend the possibilities of the equations (3) and (4) for a quantitative description of the effects of the substituents in these BNRs when they can have a single RDS. This is of considerable importance since due to the presence of the single RDS in the multistep BNRs, it provides a possibilities to modify the BNRs to achieve higher or lower rates as desired. To the best of our knowledge, this has been the first comprehensive study of the mechanistic features of the aminolysis in the BNRs so far.

\section{RESULTS AND DISCUSSION}

The key results are summarized in Table 1.

\section{Reaction Constants $\delta \Delta H^{\ddagger}$ and $\delta \Delta S^{\ddagger}$}

The values of the changes in the activation parameters, $\delta \Delta H^{\neq}$and $\delta \Delta S^{\neq}$, for the reactions of compounds 1 - 33 with amines $34-37,39,40$ and pyridines 38 in various solvents (Table 1, entries 1 $65)$ were obtained according to the general Hammettlike equation (1) using the Hammett's $\sigma$ substituent constants (Table $\mathbf{S} 1$ in the supplementary data) [65].

The $\delta \Delta H^{\ddagger}$ and $\delta \Delta S^{\neq}$values in Table 1 reflect the sensitivity of activation parameters to substituent nature in the leaving groups, nucleophiles and nonleaving groups and strongly depend on solvation of reactants and TSs $[31-36,66,67]$.

Recent analysis of the reaction constants $\delta \Delta H^{\ddagger}$ and $\delta \Delta S^{\ddagger}$ for typical BNRs has revealed a number of compensation equations that depend on the reaction rate constants and on the differences in the effects of various substituents [31]. As can be seen from the general compensation relationship, $\delta \Delta H^{\neq}$vs. $\delta \Delta S^{\neq}$(Eq. $5)$, the slope of this equation corresponds to the

$\delta \Delta H^{\neq}=\delta \Delta H_{\text {int }}^{\neq}+T_{\text {comp }} \delta \Delta S^{\neq}(5)$

compensation temperature $T_{\text {comp }}$ and their values were tested at the $\geq 95 \%$ confidence level (Table 1) [31]. Note that it is very important to test for the existence of a compensation relationship at a confidence level of $>95 \%$ [68]. The compensation temperatures $T_{\text {comp }}$ are higher than the mean experimental temperature $T_{\exp }$ excluding only entries 55-57 in Table 1 and it must be concluded that the compensation correlations are not caused by experimental errors [69]. As for the exact physical - chemical sense of the enthalpy - entropy compensation, this is still a debated question [68, 7075]. Nevertheless, when $T_{\text {comp }}>T_{\text {exp }}$, it is necessary to 


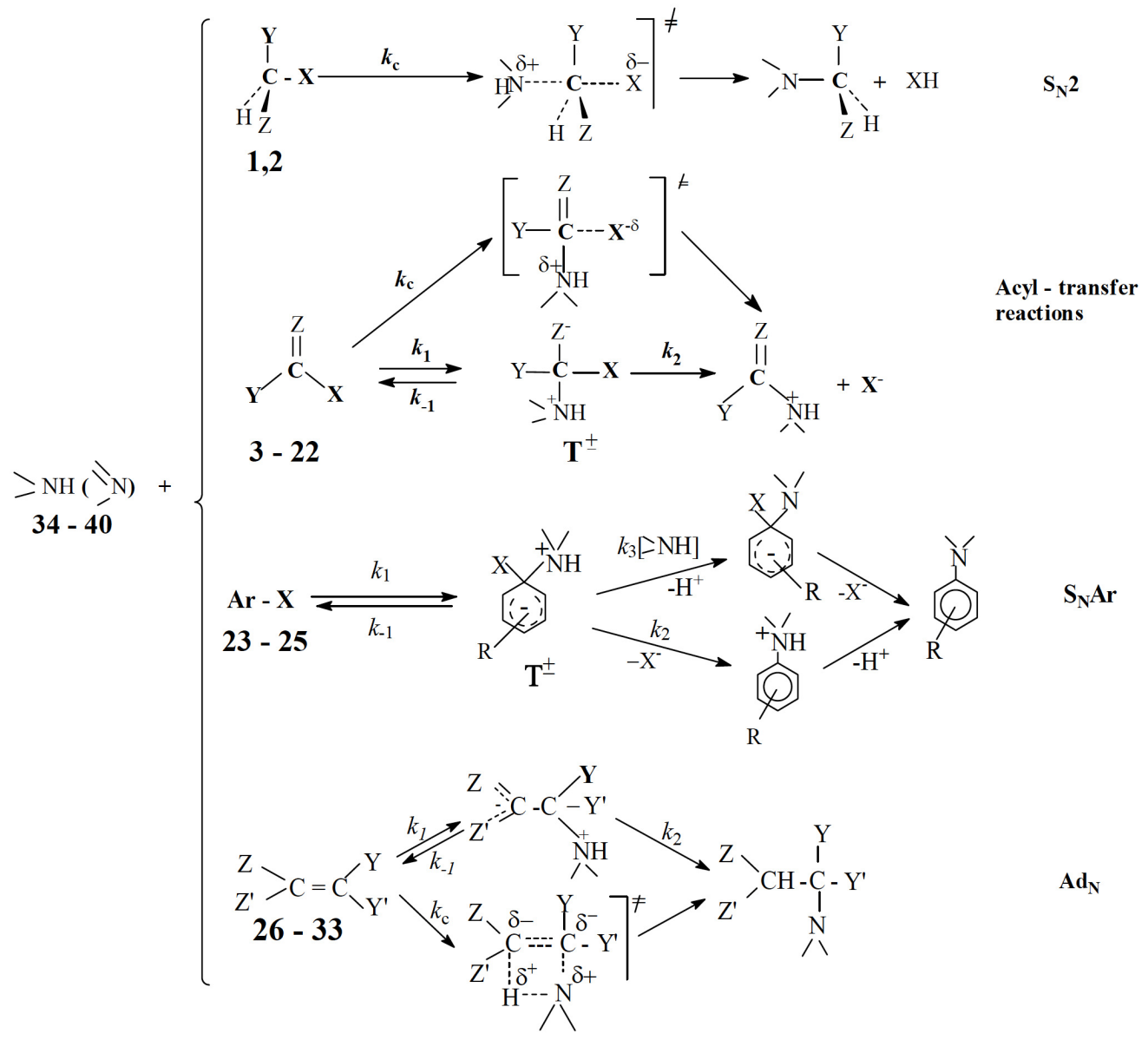

$1 \mathrm{X}=\mathrm{I}, \mathrm{Y}=\mathrm{Z}=\mathrm{H}$

$2 \mathrm{X}=\mathrm{Br}, \mathrm{Y}=\mathrm{H}, \mathrm{Z}=\mathrm{RC}_{6} \mathrm{H}_{4}$

$3 \mathrm{X}=\mathrm{RC}_{6} \mathrm{H}_{4} \mathrm{O}, \mathrm{Y}=4-\mathrm{NO}_{2} \mathrm{C}_{6} \mathrm{H}_{4}, \mathrm{Z}=\mathrm{O}$

$4 \mathrm{X}=2-\mathrm{NO}_{2}-\mathrm{RC}_{6} \mathrm{H}_{3} \mathrm{O}, \mathrm{Y}=\mathrm{Ph}, \mathrm{Z}=\mathrm{O}$

$5 \mathrm{X}=2,6-\left(\mathrm{NO}_{2}\right)_{2}-\mathrm{RC}_{6} \mathrm{H}_{2} \mathrm{O}, \mathrm{Y}=\mathrm{Ph}, \mathrm{Z}=\mathrm{O}$

$6 \mathrm{X}=\mathrm{RC}_{6} \mathrm{H}_{4} \mathrm{O}, \mathrm{Y}=\mathrm{Me}, \mathrm{Z}=\mathrm{O}$

$7 \mathrm{X}=\mathrm{RC}_{6} \mathrm{H}_{4} \mathrm{~S}, \mathrm{Y}=\mathrm{EtO}, \mathrm{Z}=\mathrm{O}$

$8 \mathrm{X}=\mathrm{RC}_{6} \mathrm{H}_{4} \mathrm{~S}, \mathrm{Y}=\mathrm{Et}, \mathrm{Z}=\mathrm{O}$

$9 \mathrm{X}=\mathrm{RC}_{6} \mathrm{H}_{4} \mathrm{~S}, \quad \mathrm{Y}=\mathrm{PhNH}, \mathrm{Z}=\mathrm{O}$

$10 \mathrm{X}=\mathrm{RC}_{6} \mathrm{H}_{4} \mathrm{O}, \mathrm{Y}=$ cyclo $-\mathrm{C}_{3} \mathrm{H}_{5}, \mathrm{Z}=\mathrm{O}$

$11 \mathrm{X}=\mathrm{RC}_{6} \mathrm{H}_{4} \mathrm{O}, \mathrm{Y}=$ cyclo- $\mathrm{C}_{4} \mathrm{H}_{7}, \mathrm{Z}=\mathrm{O}$

$12 \mathrm{X}=\mathrm{RC}_{6} \mathrm{H}_{4} \mathrm{~S}, \quad \mathrm{Y}=\mathrm{PhCH}_{2}, \mathrm{Z}=\mathrm{S}$

$13 \mathrm{X}=\mathrm{RC}_{6} \mathrm{H}_{4} \mathrm{~S}, \quad \mathrm{Y}=\mathrm{Me}, \mathrm{Z}=\mathrm{O}$

$14 \mathrm{X}=\mathrm{RC}_{6} \mathrm{H}_{4} \mathrm{~S}, \quad \mathrm{Y}=\mathrm{PhCH}_{2}, \mathrm{Z}=\mathrm{O}$

$15 \mathrm{X}=\mathrm{Cl}, \mathrm{Y}=\mathrm{Ph}, \mathrm{Z}=\mathrm{O}$

$16 \mathrm{X}=\mathrm{Cl}, \mathrm{Y}=\mathrm{PhO}, \mathrm{Z}=\mathrm{O}$

$17 \mathrm{X}=\mathrm{Cl}, \mathrm{Y}=2-\mathrm{C}_{4} \mathrm{H}_{3} \mathrm{OCH}=\mathrm{CH}-, \mathrm{Z}=\mathrm{O}$

$18 \mathrm{X}=\mathrm{Cl}, \mathrm{Y}=3-\mathrm{C}_{4} \mathrm{H}_{3} \mathrm{~S}-, \mathrm{Z}=\mathrm{O}$

$19 \mathrm{X}=2,4-\left(\mathrm{NO}_{2}\right)_{2} \mathrm{C}_{6} \mathrm{H}_{3} \mathrm{O}, \mathrm{Y}=\mathrm{Ph}, \mathrm{Z}=\mathrm{O}$

$20 \mathrm{X}=\mathrm{CH}_{2} \mathrm{Br}, \mathrm{Y}=\mathrm{RC}_{6} \mathrm{H}_{4}, \mathrm{Z}=\mathrm{O}$
$21 \mathrm{X}=4-\mathrm{NO}_{2} \mathrm{C}_{6} \mathrm{H}_{4} \mathrm{O}, \mathrm{Y}=\mathrm{RC}_{6} \mathrm{H}_{4}, \mathrm{Z}=\mathrm{O}$

$22 \mathrm{X}=2,4-\left(\mathrm{NO}_{2}\right)_{2} \mathrm{C}_{6} \mathrm{H}_{3} \mathrm{O}, \mathrm{Y}=\mathrm{RC}_{6} \mathrm{H}_{4}, \mathrm{Z}=\mathrm{O}$

$23 \mathrm{X}=\mathrm{Cl}, \mathrm{Ar}=2-\mathrm{NO}_{2}-\mathrm{RC}_{6} \mathrm{H}_{3}$

$24 \mathrm{X}=\mathrm{Cl}, \mathrm{Ar}=2,4,6-\left(\mathrm{NO}_{2}\right)_{3} \mathrm{C}_{6} \mathrm{H}_{2}$

$25 \mathrm{X}=\mathrm{Cl}, \mathrm{Ar}=\mathrm{RC}_{6} \mathrm{H}_{4}$

$26 \mathrm{PhCH}=\mathrm{C}(\mathrm{CN})_{2}$

27 (E) $-\mathrm{RC}_{6} \mathrm{H}_{4} \mathrm{CH}=\mathrm{CHNO}_{2}$

$28 \mathrm{PhC} \equiv \mathrm{C}-\mathrm{C}(\mathrm{O}) \mathrm{Ph}$

$294-\mathrm{NO}_{2} \mathrm{C}_{6} \mathrm{H}_{4} \mathrm{CH}=\mathrm{CHNO}_{2}$

$30 \mathrm{RC}_{6} \mathrm{H}_{4} \mathrm{CH}=\mathrm{C}(\mathrm{CN}) \mathrm{C}_{6} \mathrm{H}_{4} \mathrm{NO}_{2}-4$

$31 \mathrm{RC}_{6} \mathrm{H}_{4} \mathrm{CH}=\mathrm{C}(\mathrm{Ph}) \mathrm{NO}_{2}$

$32 \mathrm{RC}_{6} \mathrm{H}_{4} \mathrm{CH}=\mathrm{C}(\mathrm{COOEt})_{2}$

$33 \mathrm{RC}_{6} \mathrm{H}_{4} \mathrm{CH}=\mathrm{C}(\mathrm{COOEt}) \mathrm{COCH}_{3}$

34 Imidazole

$35 \mathrm{NH}_{2} \mathrm{CH}_{2} \mathrm{COO}^{-}$

$36 \mathrm{R}^{\prime} \mathrm{C}_{6} \mathrm{H}_{4} \mathrm{CH}_{2} \mathrm{NH}_{2}$

$37 \mathrm{R}^{\prime} \mathrm{C}_{6} \mathrm{H}_{4} \mathrm{NH}_{2}$

$38 \mathrm{R}^{\prime} \mathrm{C}_{5} \mathrm{H}_{4} \mathrm{~N}$

$39 \mathrm{NH}_{3}$

$40 \mathrm{C}_{5} \mathrm{H}_{10} \mathrm{NH}$

2 - 14, 20 -23, 25, 27, 30 - 33, 36 - 38:
a. $\mathrm{R}=4-\mathrm{NH}_{2}$
f. $\mathrm{R}=3-\mathrm{Me}$
k. $\mathrm{R}=4-\mathrm{Br}$
b. $\mathrm{R}=4-\mathrm{MeO}$
g. $\mathrm{R}=4-\mathrm{NMe}_{2}$
l. $\mathrm{R}=3-\mathrm{F}$
p. $\mathrm{R}=3-\mathrm{Br}$
u. $\mathrm{R}=4-\mathrm{NO}_{2}$
q. $\mathrm{R}=4-\mathrm{CF}_{3}$
v. $\mathrm{R}=3-\mathrm{NO}_{2}$
z. $\mathrm{R}=4-\mathrm{SO}_{2} \mathrm{NH}_{2}$
c. $\mathrm{R}=4-\mathrm{Me}$
m. $\mathrm{R}=3-\mathrm{Cl}$
r. $\mathrm{R}=3-\mathrm{CF}_{3}$
w. $\mathrm{R}=4-\mathrm{COOEt}$
d. $\mathrm{R}=3-\mathrm{NH}_{2}$
i. $\mathrm{R}=4-\mathrm{F}$
n. $\mathrm{R}=3,4-\mathrm{Cl}_{2}$
s. $\mathrm{R}=4-\mathrm{CN}$
x. $\mathrm{R}=4-\mathrm{SO}_{2} \mathrm{CH}_{3}$
j. $\mathrm{R}=4-\mathrm{Cl}$
o. $\mathrm{R}=3,5-\mathrm{Cl}_{2}$
t. $\mathrm{R}=3-\mathrm{CN}$
y. $\mathrm{R}=3-\mathrm{SO}_{2} \mathrm{CH}_{3}$
aa $\mathrm{R}=4-\mathrm{SO}_{2} \mathrm{CF}$

Scheme 1: Reactions of compounds 1 - 33 with amines $34-40$. 
Table 1: Changes in the reaction constants $\delta \Delta G^{\neq}, \delta \Delta H_{\text {int }}^{\ddagger}, \delta \Delta H^{\ddagger}$, and $\delta \Delta S^{\neq}$, the experimental and compensation temperatures $T_{\exp }$ and $T_{\text {comp }}$, rate constants of the RDS and the Hammett $\rho$ reaction constants in the reactions of compounds 1 - 33 with primary amines $35-37,39$, secondary cyclic amines 34,40 , and pyridines 38 in various solvents

\begin{tabular}{|c|c|c|c|c|c|c|c|c|c|c|c|}
\hline Entry & Reactants & Solvent & $\begin{array}{c}\delta \Delta G^{\neq} \text {I } \\
\mathrm{kJ} \mathrm{mol}^{-1} \sigma^{-1}\end{array}$ & $\begin{array}{l}\delta \Delta H_{\text {int }}^{ \pm}{ }^{\mathrm{a}} / \\
\mathrm{kJ} \mathrm{mol}_{\sigma^{-1}}^{-1}\end{array}$ & $\begin{array}{c}\delta \Delta H^{\ddagger} \mathrm{I} \\
\mathrm{kJ} \mathrm{mol}_{\sigma^{-1}}^{-1}\end{array}$ & $\begin{array}{c}\delta \Delta S^{\ddagger} I \\
\mathrm{~J} \mathrm{~mol}^{-1} \mathrm{~K}^{-1} \\
\sigma^{-1}\end{array}$ & $T_{\exp } I$ & $\underset{\mathrm{b} / \mathrm{K}}{T_{\text {comp }}}$ & $\begin{array}{l}\text { Rate } \\
\text { constant } \\
\text { of the } \\
\text { RDS }\end{array}$ & $\rho$ & Ref. $^{c}$ \\
\hline & $\begin{array}{c}\text { Substituents } R \text { are } \\
\text { Acyl-transfer reactions }\end{array}$ & varied on & leaving & groups & & & & & & & \\
\hline 1 & $\begin{array}{c}\text { 4- } \mathrm{NO}_{2} \mathrm{C}_{6} \mathrm{H}_{4} \mathrm{COOC}_{6} \mathrm{H}_{4} \mathrm{R} \\
\mathbf{3 c , h}, \mathbf{j}, \mathbf{s}, \mathbf{u}+\text { Imidazole } \mathbf{3 4}\end{array}$ & $\begin{array}{l}10 v o / \% \\
\text { MeCN- } \\
\mathrm{H}_{2} \mathrm{O}\end{array}$ & -9.1 & -9.2 & -1.1 & 25.4 & 315 & 320 & $k_{1}$ & 1.56 & $\begin{array}{l}{[31,} \\
32]\end{array}$ \\
\hline 2 & $\begin{array}{c}\mathrm{PhCOOC}_{6} \mathrm{H}_{3} \mathrm{R}_{-\mathrm{NO}_{2}-2} \\
4 \mathbf{u}, \mathbf{v} \\
+\mathrm{NH}_{2} \mathrm{CH}_{2} \mathrm{COO}^{-} 35\end{array}$ & $\begin{array}{c}60 \% \\
\text { dioxane- } \\
40 \% \mathrm{H}_{2} \mathrm{O}\end{array}$ & -7.2 & -6.5 & -16.8 & -32.1 & 298 & 320 & $k_{1}$ & 1.26 & [31] \\
\hline 3 & $\begin{array}{c}\mathrm{PhCOOC}_{6} \mathrm{H}_{2} \mathrm{R}-\left(\mathrm{NO}_{2}\right)_{2}-2,6 \\
5 \mathbf{h}, \mathbf{u} \\
+\mathrm{NH}_{2} \mathrm{CH}_{2} \mathrm{COO}^{-} 35\end{array}$ & $\begin{array}{c}60 \% \\
\text { dioxane- } \\
40 \% \mathrm{H}_{2} \mathrm{O}\end{array}$ & -6.9 & -6.2 & -16.8 & -33.1 & 298 & 320 & $k_{1}$ & 1.24 & [31] \\
\hline 4 & $\begin{array}{c}\mathrm{MeC}(\mathrm{O}) \mathrm{OC}_{6} \mathrm{H}_{4} \mathrm{R} \\
\mathbf{6 j , \mathbf { s }} \\
+4-\mathrm{MeC}_{6} \mathrm{H}_{4} \mathrm{CH}_{2} \mathrm{NH}_{2} 3 \mathbf{3 c}\end{array}$ & DMSO & -5.70 & -5.48 & -8.79 & -10.33 & 298 & 320 & $k_{2}$ & 1.61 & - \\
\hline 5 & $\begin{array}{c}\mathrm{MeC}(\mathrm{O}) \mathrm{OC}_{6} \mathrm{H}_{4} \mathrm{R} \\
\mathbf{6 j}, \mathbf{s} \\
+4-\mathrm{ClC}_{6} \mathrm{H}_{4} \mathrm{CH}_{2} \mathrm{NH}_{2} \mathbf{3 6 \mathbf { j }}\end{array}$ & DMSO & -9.31 & -9.19 & -10.85 & -5.17 & 298 & 320 & $k_{2}$ & 1.85 & - \\
\hline 6 & $\begin{array}{c}\operatorname{EtOC}(\mathrm{O}) \mathrm{SC}_{6} \mathrm{H}_{4} \mathrm{R} \\
\mathbf{7} \mathbf{c}, \mathbf{k} \\
+4-\mathrm{MeOC}_{6} \mathrm{H}_{4} \mathrm{CH}_{2} \mathrm{NH}_{2} \mathbf{3 6} \mathbf{b}\end{array}$ & $\mathrm{MeCN}$ & -10.2 & -9.82 & -19.85 & -31.35 & 308 & 320 & $k_{\mathrm{c}}$ & 1.63 & - \\
\hline 7 & $\begin{array}{c}\mathrm{EtOC}(\mathrm{O}) \mathrm{SC}_{6} \mathrm{H}_{4} \mathrm{R} \\
\mathbf{7} \mathbf{c}, \mathbf{k} \\
+4-\mathrm{ClC}_{6} \mathrm{H}_{4} \mathrm{CH}_{2} \mathrm{NH}_{2} \mathbf{3 6 \mathbf { j }}\end{array}$ & $\mathrm{MeCN}$ & -8.55 & -8.79 & -2.1 & 20.92 & 308 & 320 & $k_{\mathrm{c}}$ & 1.37 & - \\
\hline 8 & $\begin{array}{c}\mathrm{EtC}(\mathrm{O}) \mathrm{SC}_{6} \mathrm{H}_{4} \mathrm{R} \\
\mathbf{8 c}, \mathbf{m} \\
+\mathbf{4 -} \mathrm{MeOC}_{6} \mathrm{H}_{4} \mathrm{CH}_{2} \mathrm{NH}_{2} \mathbf{3 6} \mathbf{b}\end{array}$ & $\mathrm{MeCN}$ & -12.39 & -12.84 & -0.70 & 37.94 & 308 & 320 & $k_{2}$ & 2.12 & - \\
\hline 9 & $\begin{array}{c}\mathrm{PhNHC}_{(\mathrm{O}) \mathrm{SC}_{6} \mathrm{H}_{4} \mathrm{R}} \\
\mathbf{9} \mathbf{c}, \mathbf{k} \\
+ \text { 4- } \mathrm{MeOC}_{6} \mathrm{H}_{4} \mathrm{CH}_{2} \mathrm{NH}_{2} \mathbf{3 6} \mathbf{b}\end{array}$ & $\mathrm{MeCN}$ & -12.20 & -12.67 & -3.77 & 27.8 & 303 & 320 & $k_{\mathrm{c}}$ & 2.07 & - \\
\hline 10 & $\begin{array}{c}\mathrm{PhNHC}(\mathrm{O}) \mathrm{SC}_{6} \mathrm{H}_{4} \mathrm{R} \\
\mathbf{9 c}, \mathbf{k} \\
+4-\mathrm{ClC}_{6} \mathrm{H}_{4} \mathrm{CH}_{2} \mathrm{NH}_{2} \mathbf{3 6} \mathbf{j}\end{array}$ & $\mathrm{MeCN}$ & -10.70 & -11.93 & 11.50 & 73.22 & 303 & 320 & $k_{\mathrm{c}}$ & 1.74 & - \\
\hline 11 & $\begin{array}{c}\text { cyclo- } \mathrm{C}_{3} \mathrm{H}_{5} \mathrm{C}(\mathrm{O}) \mathrm{OC}_{6} \mathrm{H}_{4} \mathrm{R} \\
10 \mathrm{t}, \mathrm{u} \\
+4-\mathrm{MeC}_{6} \mathrm{H}_{4} \mathrm{CH}_{2} \mathrm{NH}_{2} 36 \mathrm{c}\end{array}$ & $\mathrm{MeCN}$ & -15.94 & $\begin{array}{c}- \\
(-16.07)\end{array}$ & 6.55 & 70.70 & 318 & - & $k_{2}$ & 2.47 & - \\
\hline 12 & $\begin{array}{c}\text { cyclo- } \mathrm{C}_{3} \mathrm{H}_{5} \mathrm{C}(\mathrm{O}) \mathrm{OC}_{6} \mathrm{H}_{4} \mathrm{R} \\
10 \mathrm{t}, \mathrm{u} \\
+3-\mathrm{ClC}_{6} \mathrm{H}_{4} \mathrm{CH}_{2} \mathrm{NH}_{2} 36 \mathrm{~m}\end{array}$ & $\mathrm{MeCN}$ & -20.45 & $\begin{array}{c}- \\
(-20.56)\end{array}$ & -3.59 & 53.03 & 318 & - & $k_{2}$ & 2.97 & - \\
\hline 13 & $\begin{array}{c}\text { cyclo- } \mathrm{C}_{4} \mathrm{H}_{7} \mathrm{C}(\mathrm{O}) \mathrm{OC}_{6} \mathrm{H}_{4} \mathrm{R} \\
11 \mathrm{t}, \mathrm{u} \\
+4-\mathrm{MeC}_{6} \mathrm{H}_{4} \mathrm{CH}_{2} \mathrm{NH}_{2} 36 \mathrm{c}\end{array}$ & $\mathrm{MeCN}$ & -15.59 & $\begin{array}{c}- \\
(-15.68)\end{array}$ & -0.59 & 47.15 & 318 & - & $k_{2}$ & 2.41 & - \\
\hline 14 & $\begin{array}{c}\text { cyclo- } \mathrm{C}_{4} \mathrm{H}_{7} \mathrm{C}(\mathrm{O}) \mathrm{OC}_{6} \mathrm{H}_{4} \mathrm{R} \\
11 \mathrm{t}, \mathrm{u} \\
+3-\mathrm{ClC}_{6} \mathrm{H}_{4} \mathrm{CH}_{2} \mathrm{NH}_{2} 36 \mathrm{~m}\end{array}$ & $\mathrm{MeCN}$ & -17.96 & $\begin{array}{c}- \\
(-18.04)\end{array}$ & -2.96 & 47.14 & 318 & - & $k_{2}$ & 3.04 & - \\
\hline 15 & $\begin{array}{c}\mathrm{PhCH}_{2} \mathrm{C}(\mathrm{S}) \mathrm{SC}_{6} \mathrm{H}_{4} \mathrm{R} \\
\mathbf{1 2 \mathbf { c } , \mathbf { k }} \\
+4-\mathrm{MeOC}_{6} \mathrm{H}_{4} \mathrm{NH}_{2} \text { 37b }\end{array}$ & $\mathrm{MeCN}$ & -15.05 & $\begin{array}{c}- \\
(-15.69)\end{array}$ & 1.05 & 52.3 & 308 & - & $k_{2}$ & 2.43 & - \\
\hline
\end{tabular}




\begin{tabular}{|c|c|c|c|c|c|c|c|c|c|c|c|}
\hline 16 & $\begin{array}{c}\mathrm{PhCH}_{2} \mathrm{C}(\mathrm{S}) \mathrm{SC}_{6} \mathrm{H}_{4} \mathrm{R} \\
\mathbf{1 2 c}, \mathbf{k} \\
+4-\mathrm{ClC}_{6} \mathrm{H}_{4} \mathrm{NH}_{2} \mathbf{3 7 m}\end{array}$ & $\mathrm{MeCN}$ & -16.95 & $\begin{array}{c}- \\
(-17.34)\end{array}$ & -7.30 & 31.37 & 308 & - & $k_{2}$ & 3.31 & - \\
\hline 17 & $\begin{array}{c}\mathrm{MeC}(\mathrm{O}) \mathrm{SC}_{6} \mathrm{H}_{4} \mathrm{R} \\
\mathbf{1 3 \mathbf { c } , \mathbf { k }} \\
+4-\mathrm{MeOC}_{6} \mathrm{H}_{4} \mathrm{CH}_{2} \mathrm{NH}_{2} \mathbf{3 6} \mathbf{b}\end{array}$ & $\mathrm{MeCN}$ & -30.02 & $\begin{array}{c}- \\
(-31.15)\end{array}$ & -1.02 & 94.15 & 308 & - & $k_{2}$ & 5.00 & - \\
\hline 18 & $\begin{array}{c}\mathrm{MeC}(\mathrm{O}) \mathrm{SC}_{6} \mathrm{H}_{4} \mathrm{R} \\
\mathbf{1 3 \mathbf { c } , \mathbf { k }} \\
+4-\mathrm{ClC}_{6} \mathrm{H}_{4} \mathrm{CH}_{2} \mathrm{NH}_{2} \text { 36j }\end{array}$ & $\mathrm{MeCN}$ & -30.10 & $\begin{array}{c}- \\
(-31.37)\end{array}$ & 2.10 & 104.60 & 308 & - & $k_{2}$ & 5.42 & - \\
\hline 19 & $\begin{array}{c}\mathrm{PhCH}_{2} \mathrm{C}(\mathrm{O}) \mathrm{SC}_{6} \mathrm{H}_{4} \mathrm{R} \\
\mathbf{1 4 \mathbf { c } , \mathbf { k }} \\
+4-\mathrm{MeOC}_{6} \mathrm{H}_{4} \mathrm{CH}_{2} \mathrm{NH}_{2} \mathbf{3 6} \mathbf{b}\end{array}$ & $\mathrm{MeCN}$ & -9.31 & -9.42 & 7.32 & 52.30 & 318 & 320 & $k_{2}$ & 1.42 & - \\
\hline \multirow[t]{2}{*}{20} & $\begin{array}{c}\mathrm{PhCH}_{2} \mathrm{C}(\mathrm{O}) \mathrm{SC}_{6} \mathrm{H}_{4} \mathrm{R} \\
\mathbf{1 4 \mathbf { c } , \mathbf { k }} \\
+3-\mathrm{ClC}_{6} \mathrm{H}_{4} \mathrm{CH}_{2} \mathrm{NH}_{2} \mathbf{3 6 m}\end{array}$ & $\mathrm{MeCN}$ & -13.0 & -13.17 & 13.60 & 83.67 & 318 & 320 & $k_{2}$ & 1.79 & - \\
\hline & $\begin{array}{c}\text { Substituents } R \text { are } \\
S_{N} 2 \text { reactions }\end{array}$ & varied on & nucleophiles & & & & & & & & \\
\hline 21 & $\begin{array}{l}\mathrm{R}^{\prime} \mathrm{C}_{5} \mathrm{H}_{4} \mathrm{~N} \\
38 \mathrm{~h}, \mathrm{~m}, \mathbf{o , p}, \mathbf{s} \\
+ \text { Mel } 1\end{array}$ & $\mathrm{MeCN}$ & 13.1 & 13.9 & 9.9 & -10.7 & 298 & 370 & $k_{\mathrm{c}}$ & -2.27 & $\begin{array}{c}{[31,} \\
33,34]\end{array}$ \\
\hline \multirow[t]{2}{*}{22} & 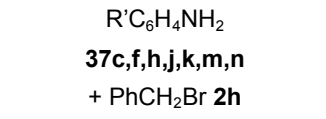 & $\mathrm{EtOH}$ & 5.6 & 5.1 & 8.0 & 7.9 & 303 & 370 & $k_{\mathrm{c}}$ & -0.89 & $\begin{array}{c}{[31,} \\
33,34]\end{array}$ \\
\hline & Acyl-transfer reactions & & & & & & & & & & \\
\hline 23 & $\begin{array}{c}\mathrm{R}^{\prime} \mathrm{C}_{6} \mathrm{H}_{4} \mathrm{NH}_{2} \\
37 \mathbf{c}, \mathbf{h}, \mathbf{j}, \mathbf{u}, \mathbf{v} \\
+\mathrm{PhC}(\mathrm{O}) \mathrm{Cl} 15\end{array}$ & $\mathrm{C}_{6} \mathrm{H}_{6}$ & 14.7 & 14.7 & 14.7 & 0 & 298 & 370 & $k_{1}$ & -2.90 & $\begin{array}{c}{[31,} \\
32,34]\end{array}$ \\
\hline 24 & $\begin{array}{c}\mathrm{R}^{\prime} \mathrm{C}_{6} \mathrm{H}_{4} \mathrm{NH}_{2} \\
\mathbf{3 7 h , m , \mathbf { v }} \\
+\mathrm{PhOC}(\mathrm{O}) \mathrm{Cl} \mathbf{1 6 h}\end{array}$ & $\mathrm{MeCN}$ & 11.8 & 11.0 & 15.3 & 11.6 & 298 & 370 & $k_{1}$ & -2.22 & $\begin{array}{c}{[31,} \\
32,34]\end{array}$ \\
\hline 25 & 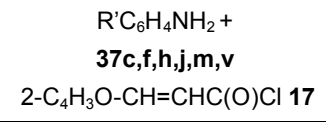 & $\mathrm{C}_{6} \mathrm{H}_{6}$ & 18.1 & 18.6 & 16.2 & -6.4 & 298 & 370 & $k_{1}$ & -2.86 & $\begin{array}{l}{[31,} \\
34]\end{array}$ \\
\hline 26 & $\begin{array}{c}\mathrm{R}^{\prime} \mathrm{C}_{6} \mathrm{H}_{4} \mathrm{NH}_{2}+ \\
\text { 37b,c,f,h,j,m, } \mathbf{v} \\
3-\mathrm{C}_{4} \mathrm{H}_{3} \mathrm{~S}-\mathrm{C}(\mathrm{O}) \mathrm{Cl} 18\end{array}$ & $\mathrm{C}_{6} \mathrm{H}_{6}$ & 18.1 & 18.2 & 17.5 & -2.0 & 298 & 370 & $k_{1}$ & -3.21 & $\begin{array}{l}{[31,} \\
34]\end{array}$ \\
\hline 27 & $\begin{array}{c}\mathrm{R}^{\prime} \mathrm{C}_{5} \mathrm{H}_{4} \mathrm{~N} \\
\mathbf{3 8 f}, \mathbf{g} \\
+\mathrm{PhC}(\mathrm{O}) \mathrm{OC}_{6} \mathrm{H}_{3}\left(\mathrm{NO}_{2}\right)_{2}-2,4 \\
\mathbf{1 9}\end{array}$ & $\begin{array}{c}44 \mathrm{wt} \% \\
\mathrm{EtOH}- \\
\mathrm{H}_{2} \mathrm{O}\end{array}$ & 25.61 & 18.48 & 57.87 & 106.47 & 303 & 370 & $k_{2}$ & -5.26 & $\begin{array}{l}{[36,} \\
64]\end{array}$ \\
\hline 28 & $\begin{array}{c}\mathrm{MeC}(\mathrm{O}) \mathrm{OC}_{6} \mathrm{H}_{4} \mathrm{Cl}-4 \\
\underset{6 \mathbf{j}}{+} \\
+\mathrm{R}^{\prime} \mathrm{C}_{6} \mathrm{H}_{4} \mathrm{CH}_{2} \mathrm{NH}_{2} \mathbf{3 6}, \mathbf{j}\end{array}$ & DMSO & 5.20 & $\begin{array}{c}- \\
(6.69)\end{array}$ & -1.05 & -20.92 & 298 & - & $k_{2}$ & -1.05 & - \\
\hline 29 & $\begin{array}{c}\mathrm{MeC}(\mathrm{O}) \mathrm{OC}_{6} \mathrm{H}_{4} \mathrm{CN}-4 \\
\quad \mathbf{6 s} \\
+\mathrm{R}^{\prime} \mathrm{C}_{6} \mathrm{H}_{4} \mathrm{CH}_{2} \mathrm{NH}_{2} 3 \mathbf{3 6}, \mathbf{j}\end{array}$ & DMSO & -2.12 & $\begin{array}{c}- \\
(-1.35)\end{array}$ & -5.22 & -10.47 & 298 & - & $k_{2}$ & -0.56 & - \\
\hline 30 & $\begin{array}{c}\mathrm{PhNHC}(\mathrm{O}) \mathrm{SC}_{6} \mathrm{H}_{4} \mathrm{Me}-4 \\
9 \mathrm{c} \\
+\mathrm{R}^{\prime} \mathrm{C}_{6} \mathrm{H}_{4} \mathrm{CH}_{2} \mathrm{NH}_{2} \mathbf{3 6} \mathbf{b}, \mathbf{j}\end{array}$ & $\mathrm{MeCN}$ & 7.62 & 10.85 & -7.06 & -48.4 & 303 & 370 & $k_{\mathrm{c}}$ & -1.30 & - \\
\hline 31 & $\begin{array}{c}\mathrm{PhNHC}(\mathrm{O}) \mathrm{SC}_{6} \mathrm{H}_{4} \mathrm{Br}-4 \\
\mathbf{9 k} \\
+\mathrm{R}^{\prime} \mathrm{C}_{6} \mathrm{H}_{4} \mathrm{CH}_{2} \mathrm{NH}_{2} \mathbf{3 6} \mathbf{b}, \mathbf{j}\end{array}$ & $\mathrm{MeCN}$ & 8.82 & 9.62 & 5.16 & -12.06 & 303 & 370 & $k_{\mathrm{c}}$ & -1.54 & - \\
\hline 32 & $\begin{array}{c}\text { cyclo- } \mathrm{C}_{3} \mathrm{H}_{5} \mathrm{C}(\mathrm{O}) \mathrm{OC}_{6} \mathrm{H}_{4} \mathrm{CN}-3 \\
10 \mathrm{t} \\
+\mathrm{R}^{\prime} \mathrm{C}_{6} \mathrm{H}_{4} \mathrm{CH}_{2} \mathrm{NH}_{2} 36 \mathbf{c}, \mathbf{m}\end{array}$ & $\mathrm{MeCN}$ & 12.83 & 13.64 & 7.91 & -15.50 & 318 & 370 & $k_{2}$ & -2.10 & - \\
\hline
\end{tabular}


(Table 1). Continued.

\begin{tabular}{|c|c|c|c|c|c|c|c|c|c|c|c|}
\hline 33 & $\begin{array}{c}\text { cyclo- } \mathrm{C}_{3} \mathrm{H}_{5} \mathrm{C}(\mathrm{O}) \mathrm{OC}_{6} \mathrm{H}_{4} \mathrm{NO}_{2}- \\
4 \\
10 \mathrm{u} \\
+\mathrm{R}^{\prime} \mathrm{C}_{6} \mathrm{H}_{4} \mathrm{CH}_{2} \mathrm{NH}_{2} 36 \mathrm{c}, \mathrm{m}\end{array}$ & $\mathrm{MeCN}$ & 6.91 & 8.91 & -5.42 & -38.74 & 318 & 370 & $k_{2}$ & -1.36 & - \\
\hline 34 & $\begin{array}{c}\text { cyclo- } \mathrm{C}_{4} \mathrm{H}_{7} \mathrm{C}(\mathrm{O}) \mathrm{OC}_{6} \mathrm{H}_{4} \mathrm{CN}-3 \\
11 \mathrm{t} \\
+\mathrm{R}^{\prime} \mathrm{C}_{6} \mathrm{H}_{4} \mathrm{CH}_{2} \mathrm{NH}_{2} 36 \mathbf{c}, \mathbf{m}\end{array}$ & $\mathrm{MeCN}$ & 12.68 & 13.49 & 7.76 & -15.48 & 318 & 370 & $k_{2}$ & -2.08 & - \\
\hline 35 & $\begin{array}{c}\text { cyclo- } \mathrm{C}_{4} \mathrm{H}_{7} \mathrm{C}(\mathrm{O}) \mathrm{OC}_{6} \mathrm{H}_{4} \mathrm{NO}_{2}-4 \\
11 \mathrm{u} \\
+\mathrm{R}^{\prime} \mathrm{C}_{6} \mathrm{H}_{4} \mathrm{CH}_{2} \mathrm{NH}_{2} 36 \mathrm{c}, \mathrm{m}\end{array}$ & $\mathrm{MeCN}$ & 9.57 & 10.38 & 4.65 & -15.5 & 318 & 370 & $k_{2}$ & -1.36 & - \\
\hline 36 & $\begin{array}{c}\mathrm{MeC}(\mathrm{O}) \mathrm{SC}_{6} \mathrm{H}_{4} \mathrm{Me}-4 \\
\text { 13c } \\
+\mathrm{R}^{\prime} \mathrm{C}_{6} \mathrm{H}_{4} \mathrm{CH}_{2} \mathrm{NH}_{2} \mathbf{3 6} \mathbf{b}, \mathbf{j}\end{array}$ & $\mathrm{MeCN}$ & 8.42 & 8.95 & 5.86 & -8.36 & 308 & 370 & $k_{2}$ & -1.65 & - \\
\hline 37 & $\begin{array}{c}\mathrm{MeC}(\mathrm{O}) \mathrm{SC}_{6} \mathrm{H}_{4} \mathrm{Br}-4 \\
13 \mathbf{k} \\
+\mathrm{R}^{\prime} \mathrm{C}_{6} \mathrm{H}_{4} \mathrm{CH}_{2} \mathrm{NH}_{2} \mathbf{3 6} \mathbf{b}, \mathbf{j}\end{array}$ & $\mathrm{MeCN}$ & 8.36 & 8.36 & 8.36 & 0 & 308 & 370 & $k_{2}$ & -1.25 & - \\
\hline 38 & $\begin{array}{l}\mathrm{PhCH}_{2} \mathrm{C}(\mathrm{O}) \mathrm{SC}_{6} \mathrm{H}_{4} \mathrm{Me}-4 \\
14 \mathrm{c} \\
+\mathrm{R}^{\prime} \mathrm{C}_{6} \mathrm{H}_{4} \mathrm{CH}_{2} \mathrm{NH}_{2} \mathbf{3 6} \mathbf{b}, \mathbf{m}\end{array}$ & $\mathrm{MeCN}$ & 9.21 & 11.25 & -3.26 & -39.22 & 318 & 370 & $k_{2}$ & -1.54 & - \\
\hline \multirow[t]{2}{*}{39} & $\begin{array}{c}\mathrm{PhCH}_{2} \mathrm{C}(\mathrm{O}) \mathrm{SC}_{6} \mathrm{H}_{4} \mathrm{Br}-4 \\
\mathbf{1 4 k} \\
+\mathrm{R}^{\prime} \mathrm{C}_{6} \mathrm{H}_{4} \mathrm{CH}_{2} \mathrm{NH}_{2} \mathbf{3 6} \mathbf{b}, \mathbf{m}\end{array}$ & $\mathrm{MeCN}$ & 6.89 & 7.92 & 0.66 & -19.61 & 318 & 370 & $k_{2}$ & -1.44 & - \\
\hline & \multicolumn{11}{|l|}{$\mathrm{S}_{\mathrm{N}} \mathrm{Ar}$ reactions } \\
\hline 40 & 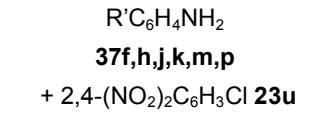 & EtOH & 18.4 & 15.4 & 37.7 & 60.4 & 318 & 370 & $k_{1}$ & -3.06 & $\begin{array}{l}{[31,} \\
34]\end{array}$ \\
\hline \multirow[t]{2}{*}{41} & $\begin{array}{c}\mathrm{R}^{\prime} \mathrm{C}_{6} \mathrm{H}_{4} \mathrm{NH}_{2} \\
\mathbf{3 7 h}, \mathbf{j}, \mathbf{m} \\
+2,4,6-\left(\mathrm{NO}_{2}\right)_{3} \mathrm{C}_{6} \mathrm{H}_{2} \mathrm{Cl} \mathbf{2 4}\end{array}$ & $\mathrm{C}_{6} \mathrm{H}_{6}$ & 27.6 & 27.7 & 27.0 & -2.0 & 298 & 370 & $k_{1}$ & -4.79 & $\begin{array}{l}{[31,} \\
34]\end{array}$ \\
\hline & \multicolumn{11}{|l|}{$\mathrm{Ad}_{\mathrm{N}}$ reactions } \\
\hline 42 & $\begin{array}{c}\mathrm{R}^{\prime} \mathrm{C}_{6} \mathrm{H}_{4} \mathrm{CH}_{2} \mathrm{NH}_{2} \\
\text { 36b-f, h-m,q,v } \\
+\mathrm{PhCH}=\mathrm{C}(\mathrm{CN})_{2} \mathbf{2 6}\end{array}$ & $\mathrm{MeCN}$ & 7.5 & 3.7 & 21.8 & 48.8 & 293 & 370 & $k_{1}$ & -0.99 & $\begin{array}{l}{[31,} \\
34]\end{array}$ \\
\hline 43 & $\begin{array}{c}\mathrm{R}^{\prime} \mathrm{C}_{6} \mathrm{H}_{4} \mathrm{NH}_{2} \\
\text { 37b-f, } \mathbf{h}, \mathbf{j}, \mathbf{k}, \mathbf{m}, \mathbf{p}-\mathbf{v} \\
+(\mathrm{E})-\mathrm{PhCH}=\mathrm{CHNO}_{2} \text { 27h }\end{array}$ & $\mathrm{MeCN}$ & 10.6 & 9.3 & 16.0 & 18.1 & 298 & 370 & $k_{1}$ & -1.88 & $\begin{array}{l}{[31,} \\
34]\end{array}$ \\
\hline 44 & $\begin{array}{c}\mathrm{R}^{\prime} \mathrm{C}_{6} \mathrm{H}_{4} \mathrm{NH}_{2} \\
\text { 37a-f, } \mathbf{h}, \mathbf{j}, \mathbf{k} \\
+\mathrm{PhCH} \equiv \mathrm{C}(\mathrm{O}) \mathrm{Ph} \mathbf{2 8}\end{array}$ & $95 \% \mathrm{EtOH}$ & 10.6 & 13.1 & -3.4 & -44.7 & 313 & 370 & $k_{1}$ & -2.18 & $\begin{array}{l}{[31,} \\
34]\end{array}$ \\
\hline 45 & $\begin{array}{c}\mathrm{R}^{\prime} \mathrm{C}_{6} \mathrm{H}_{4} \mathrm{CH}_{2} \mathrm{NH}_{2} \\
\mathbf{3 6 \mathbf { b } , \mathbf { j }} \\
+4-\mathrm{NO}_{2} \mathrm{C}_{6} \mathrm{H}_{4} \mathrm{CH}=\mathrm{CHNO}_{2} \\
\mathbf{2 9}\end{array}$ & $\mathrm{MeCN}$ & 8.38 & 10.13 & 0.84 & -25.1 & 298 & 370 & $k_{\mathrm{c}}$ & -1.55 & - \\
\hline 46 & $\begin{array}{c}\mathrm{R}^{\prime} \mathrm{C}_{6} \mathrm{H}_{4} \mathrm{CH}_{2} \mathrm{NH}_{2} \\
\mathbf{3 6 \mathbf { b } , \mathbf { j }} \\
+4-\mathrm{BrC}_{6} \mathrm{H}_{4} \mathrm{CH}=\mathrm{C}(\mathrm{CN})- \\
\mathrm{C}_{6} \mathrm{H}_{4} \mathrm{NO}_{2}-4 \\
\mathbf{3 0 k}\end{array}$ & $\mathrm{MeCN}$ & 8.32 & 8.96 & 5.86 & -8.38 & 298 & 370 & $k_{\mathrm{c}}$ & -1.30 & - \\
\hline 47 & $\begin{array}{c}\mathrm{R}^{\prime} \mathrm{C}_{6} \mathrm{H}_{4} \mathrm{CH}_{2} \mathrm{NH}_{2} \\
\mathbf{3 6 b}, \mathbf{j} \\
+4-\mathrm{MeOC}_{6} \mathrm{H}_{4} \mathrm{CH}=\mathrm{C}(\mathrm{CN})- \\
\mathrm{C}_{6} \mathrm{H}_{4} \mathrm{NO}_{2}-4 \\
\mathbf{3 0 b}\end{array}$ & $\mathrm{MeCN}$ & 6.52 & 8.45 & -0.84 & -25.1 & 298 & 370 & $k_{\mathrm{c}}$ & -0.95 & - \\
\hline
\end{tabular}




\begin{tabular}{|c|c|c|c|c|c|c|c|c|c|c|c|}
\hline 48 & $\begin{array}{c}\mathrm{RC}_{6} \mathrm{H}_{4} \mathrm{CH}_{2} \mathrm{Br} \\
\mathbf{2 h}, \mathbf{j}, \mathbf{u} \\
+\mathrm{PhNH}_{2} \\
\mathbf{3 7 h}\end{array}$ & $\mathrm{MeCN}$ & 3.3 & 4.7 & -2.0 & -17.3 & 308 & 380 & $k_{\mathrm{c}}$ & -0.55 & $\begin{array}{c}{[31,} \\
33,35]\end{array}$ \\
\hline 49 & \multicolumn{11}{|l|}{ Acyl-transfer reactions } \\
\hline 50 & $\begin{array}{c}\mathrm{RC}_{6} \mathrm{H}_{4} \mathrm{OC}(\mathrm{O}) \mathrm{Cl} \mathbf{1 6} \mathbf{b}, \mathbf{u} \\
+\mathrm{C}_{5} \mathrm{H}_{5} \mathrm{~N} \mathbf{3 8} \mathbf{h}\end{array}$ & $\mathrm{MeCN}$ & -5.92 & -6.21 & 1.20 & 23.89 & 288 & $310^{c}$ & $k_{1}$ & 1.03 & [36] \\
\hline 51 & $\begin{array}{c}\mathrm{RC}_{6} \mathrm{H}_{4} \mathrm{C}(\mathrm{O}) \mathrm{CH}_{2} \mathrm{Br} \\
\quad \mathbf{2 0 b}, \mathbf{u} \\
+3-\mathrm{MeC}_{5} \mathrm{H}_{4} \mathrm{~N} \mathbf{3 8 f} \\
\end{array}$ & $\mathrm{MeCN}$ & -1.89 & $\begin{array}{c}- \\
(-2.12)\end{array}$ & -11.03 & -28.74 & 318 & - & $k_{1}$ & 0.28 & [36] \\
\hline 53 & $\begin{array}{c}\mathrm{RC}_{6} \mathrm{H}_{4} \mathrm{COOC}_{6} \mathrm{H}_{4} \mathrm{NO}_{2}-4 \\
\mathbf{2 1 c}, \mathbf{h}, \mathbf{j}, \mathbf{s}, \mathbf{u} \\
+ \text { Imidazole } \mathbf{3 4}\end{array}$ & $\begin{array}{c}10 \text { vol\% } \\
\mathrm{MeCN}- \\
\mathrm{H}_{2} \mathrm{O}\end{array}$ & -6.8 & -6.8 & -5.6 & 3.9 & 308 & $310^{c}$ & $k_{1}$ & 1.10 & $\begin{array}{l}{[31,} \\
32]\end{array}$ \\
\hline \multirow[t]{2}{*}{54} & $\begin{array}{c}\mathrm{RC}_{6} \mathrm{H}_{4} \mathrm{C}(\mathrm{O}) \mathrm{OC}_{6} \mathrm{H}_{3}\left(\mathrm{NO}_{2}\right)_{2}-2,4 \\
\mathbf{2 2 c}, \mathbf{h}, \mathbf{j}, \mathbf{s}, \mathbf{u} \\
+ \text { Imidazole } \mathbf{3 4}\end{array}$ & $\begin{array}{c}10 \text { vol\% } \\
\mathrm{MeCN}- \\
\mathrm{H}_{2} \mathrm{O}\end{array}$ & -10.0 & -10.2 & -2.9 & 23.7 & 298 & $310^{c}$ & $k_{1}$ & 1.74 & $\begin{array}{l}{[31,} \\
32]\end{array}$ \\
\hline & \multicolumn{11}{|l|}{$S_{N} A r$ reactions } \\
\hline 55 & $\begin{array}{c}\mathrm{RC}_{6} \mathrm{H}_{4} \mathrm{Cl} \mathbf{2 5 r}, \mathbf{u}, \mathbf{x}, \mathbf{z} \\
+\mathrm{NH}_{3} 39\end{array}$ & $\mathrm{H}_{2} \mathrm{O}$ & -58.1 & -58.0 & -43.4 & 31.0 & 473 & 450 & $k_{1}$ & 8.6 & $\begin{array}{l}{[31,} \\
35]\end{array}$ \\
\hline 58 & \multicolumn{11}{|l|}{$\mathrm{Ad}_{\mathrm{N}}$ reactions } \\
\hline 59 & $\begin{array}{c}\mathrm{RC}_{6} \mathrm{H}_{4} \mathrm{CH}=\mathrm{C}(\mathrm{Ph}) \mathrm{NO}_{2} \\
\mathbf{3 1 \mathbf { b } , \mathbf { k }} \\
+4-\mathrm{MeOC}_{6} \mathrm{H}_{4} \mathrm{CH}_{2} \mathrm{NH}_{2} \\
\text { 36b }\end{array}$ & $\mathrm{MeCN}$ & -6.38 & -6.94 & 0.84 & 25.1 & 298 & 310 & $k_{\mathrm{c}}{ }^{\mathrm{d}}$ & 1.46 & - \\
\hline 60 & $\begin{array}{c}\mathrm{RC}_{6} \mathrm{H}_{4} \mathrm{CH}=\mathrm{C}(\mathrm{Ph}) \mathrm{NO}_{2} \\
\mathbf{3 1 \mathbf { b } , \mathbf { k }} \\
+4-\mathrm{ClC}_{6} \mathrm{H}_{4} \mathrm{CH}_{2} \mathrm{NH}_{2} \\
\mathbf{3 6 \mathbf { j }}\end{array}$ & $\mathrm{MeCN}$ & -6.38 & -6.64 & -1.66 & 16.72 & 298 & 310 & $k_{\mathrm{c}}{ }^{d}$ & 1.19 & - \\
\hline 61 & $\begin{array}{c}\mathrm{RC}_{6} \mathrm{H}_{4} \mathrm{CH}=\mathrm{CHNO}_{2} \\
\mathbf{2 7} \mathbf{c}, \mathbf{u} \\
+4-\mathrm{MeOC}_{6} \mathrm{H}_{4} \mathrm{CH}_{2} \mathrm{NH}_{2} \\
\mathbf{3 6 \mathbf { b }}\end{array}$ & $\mathrm{MeCN}$ & -10.13 & -10.53 & 1.76 & 39.64 & 288 & 310 & $k_{c}{ }^{d}$ & 1.82 & - \\
\hline 62 & $\begin{array}{c}\mathrm{RC}_{6} \mathrm{H}_{4} \mathrm{CH}=\mathrm{C}(\mathrm{CN})-\mathrm{C}_{6} \mathrm{H}_{4} \mathrm{NO}_{2-} \\
4 \mathbf{3 0 b}, \mathbf{k} \\
+4-\mathrm{MeOC}_{6} \mathrm{H}_{4} \mathrm{CH}_{2} \mathrm{NH}_{2} \\
\mathbf{3 6 \mathbf { b }}\end{array}$ & $\mathrm{MeCN}$ & -6.58 & -6.87 & -1.68 & 16.74 & 298 & 310 & $k_{\mathrm{c}}{ }^{\mathrm{d}}$ & 1.19 & - \\
\hline 63 & $\begin{array}{c}\mathrm{RC}_{6} \mathrm{H}_{4} \mathrm{CH}=\mathrm{C}(\mathrm{CN})-\mathrm{C}_{6} \mathrm{H}_{4} \mathrm{NO}_{2-} \\
4 \mathbf{3 0 b}, \mathbf{k} \\
+4-\mathrm{ClC}_{6} \mathrm{H}_{4} \mathrm{CH}_{2} \mathrm{NH}_{2} \\
\mathbf{3 6 \mathbf { j }}\end{array}$ & $\mathrm{MeCN}$ & -4.78 & -5.35 & 5.02 & 33.46 & 298 & 310 & $k_{\mathrm{c}}{ }^{\mathrm{d}}$ & 0.84 & - \\
\hline
\end{tabular}


(Table 1). Continued.

\begin{tabular}{|c|c|c|c|c|c|c|c|c|c|c|c|}
\hline 64 & $\begin{array}{c}\mathrm{RC}_{6} \mathrm{H}_{4} \mathrm{CH}=\mathrm{C}(\mathrm{COOEt})_{2} \mathbf{3 2} \mathbf{b}, \mathbf{k} \\
+4-\mathrm{MeOC}_{6} \mathrm{H}_{4} \mathrm{CH}_{2} \mathrm{NH}_{2} \\
\mathbf{3 6 b}\end{array}$ & $\mathrm{MeCN}$ & -7.30 & -7.85 & 2.52 & 33.48 & 293 & 310 & $k_{\mathrm{c}}{ }^{d}$ & 1.24 & - \\
\hline 65 & 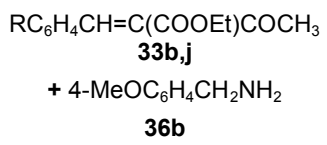 & $\mathrm{MeCN}$ & -6.30 & -7.04 & 3.34 & 33.48 & 288 & 310 & $k_{c}{ }^{d}$ & 1.03 & - \\
\hline
\end{tabular}

${ }^{a}$ Values in parentheses were calculated by Eq. $8 .{ }^{b}$ Values are taken from the reference [31]. ${ }^{c}$ The references relate to the values of $\delta \Delta G^{\ddagger}, \delta \Delta H^{\ddagger}$ int, $, \delta \Delta H^{\ddagger}, \delta \Delta S^{\ddagger}, T_{\text {exp }}$ the rate constants of the RDS, and $\rho$; if the reference is not indicated, these values are taken from Tables $1 \mathbf{S}$ and $2 \mathbf{S}$ in the supplementary data. ${ }^{\alpha}$ alue of $T_{\text {comp }}=310$ (entries $50,53,54$ and $59-65)$ is calculated by the compensation equation $\delta \Delta H^{\neq}=(-9.21 \pm 0.53)+(0.31 \pm 0.02) \delta \Delta S^{\neq}(r=0.975, s=3.0, n=23$ at the $97 \%$ confidence limits), where the $\delta \Delta H^{\neq}$and $\delta \Delta S^{\neq}$are taken from [31] and this Table.

accept the existence of a real correlation between the values of $\delta \Delta \mathrm{H}^{\neq}$and $\delta \Delta \mathrm{S}^{\neq}[69]$.

It should be noted that the intercept in Eq. 5 is the internal enthalpy constant $\delta \Delta H^{\neq}$int for the given reaction series. The latter were calculated by Eqs. 5 and 6 where the the external enthalpy constant $\delta \Delta H^{\neq}$ext depends only on the solvation effects $[31,37,38]$.

$\delta \Delta H_{\text {ext }}^{\ddagger}=T_{\text {comp }} \delta \Delta S^{\ddagger}$

However, there are the deviations from Eq. 5 for the acyl-transfer reactions (entries 11-18, 28, 29, 51 and 52 in Table 1). Obviously, in these cases $\delta \Delta H^{t}$ int values cannot be calculated by Eqs. 5 and 6 ; therefore, they were calculated by Eq. 8 .

\section{Reaction Constants $\delta \Delta G^{\neq}$and $\delta \Delta H_{\text {int }}^{\ddagger}$}

The changes in the free energy of activation reaction constant, $\delta \Delta G^{\neq}$, reflect a substituent effect in the leaving groups, nucleophiles, and nonleaving groups. The values of $\delta \Delta G^{\neq}$are negative for the reaction series in which the substituents $R$ are varied in the leaving and nonleaving groups (entries $1-20$ and $50-65$ in Table 1) excluding entries 48 and 49 for $S_{N} 2$ reactions of substituted benzyl bromide with aniline and pyridine, respectively $[9,20,31]$. On the other hand, reaction series in which change is only made to substituent $R^{\prime}$ in the nucleophile (entries 21 - 47) are characterized by positive value of $\delta \Delta G^{\neq}$. Such variation in the signs of the $\delta \Delta G^{\neq}$values is common for the BNRs [31-36] according to the Hammett-like equation $\delta \Delta G^{\neq}=-2.303 R T_{\exp \rho}[66]$.

The dependence $\delta \Delta G^{\neq}$vs. $\rho$ has been developed for these reactions (Eq. 7) [31]. However, Eq. 7 does not speak about peculiarities of the mechanisms of the BNRs because the Hammett $\rho$ values may depend on the contributions of each step in a stepwise process and the TS structure in the concerted reaction (Scheme 1) [31]. $\delta \Delta \mathrm{G}^{\neq}=0.04-6.04 \rho$

$r=0.995, s=1.56, n=98$

As follows from the data in Table 1 , the reaction constants $\delta \Delta H^{ \pm}$int and $\delta \Delta G^{\neq}$are close for the majority of reaction series, that is, $\delta \Delta G^{\neq} \approx \delta \Delta H^{*}$ int (cf. ref. 25). A single linear relationship between $\delta \Delta H^{\neq}$int and $\delta \Delta G^{\neq}$for the BNRs (entries $1-10,19-26,30-50,53-65$ in Table 1) has been developed (Eq. 8) (Figure 1).

$\delta \Delta H_{\text {int }}^{\neq}=(0.05 \pm 0.17)+(1.01 \pm 0.01) \delta \Delta G^{\neq}$

$r=0.996, s=1.25, n=52$

The intercept in Eq. 8 corresponds to the $\delta \Delta G_{\text {ext }}^{\neq}$ value and $\delta \Delta G_{\text {ext }}^{\neq}=\delta \Delta H_{\text {ext }}^{\neq}-T_{\text {ext }} \delta \Delta S_{\text {ext }}^{\neq} \approx 0[31,66,76]$. It is noteworthy that Eq. 8 coincides practically with the similar Eq. 3 relating to the BNRs for which the $k_{\mathrm{c}}$ and $k_{1}$ steps were rate-determining (Scheme 1) [31]. Therefore, a single Hammett-like linear Eq. 8 between the $\delta \Delta H^{\neq}$int and $\delta \Delta G^{\neq}$values for the BNRs can be feasible provided that steps with $k_{\mathrm{c}}, k_{1}$ and $k_{2}$ (Scheme 1) are presumed to be single rate-determining. In this case, Eq. 8 can be taken as an evidence for a shift of the RDS in the multistep BNRs.

There is the one deviation from the dependence depicted in Eq. 8 for the reactions of entry 27 in Table 1. This deviation can be explained by a larger change of activation entropy due to the stronger difference in solvation of TS forming at a breakdown of $\mathrm{T}^{ \pm}$for acyltransfer reactions (step $k_{2}$ in Scheme 1) [36,64].

\section{Mechanistic Criteria for the Aminolysis in the BNRs}

To determine the single RDS for the aminolysis in the BNRs discussed (entries $1-65$ in Table 1) the analysis of the kinetic data was made on the basis of the Brønsted and Hammett equations (Table $2 \mathrm{~S}$ in the supplementary data) [40-53,64,77-88]. It should be noted that the biphasic Brønsted plots found for the aminolysis of compounds 6, 8, $10-14,19,20$ (entries $4,5,8,11-20,27-29,32-39,52$ in Tables 1 and 


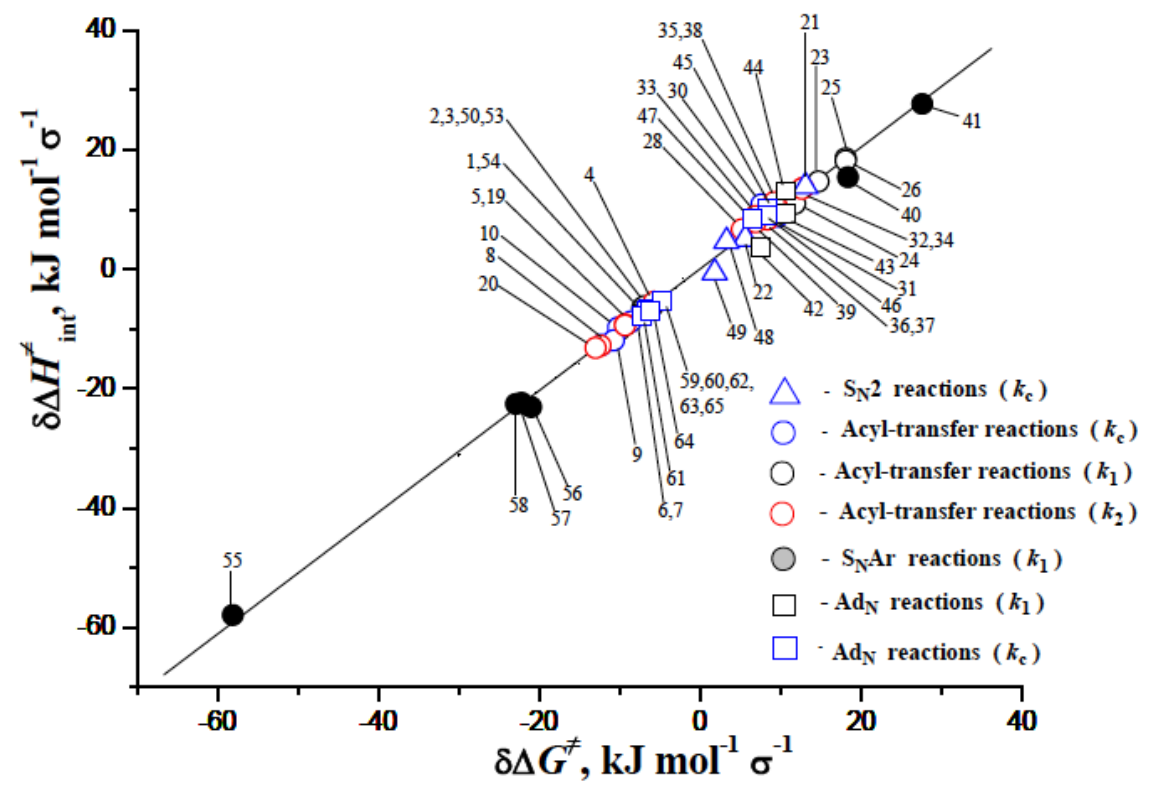

Figure 1: Plot of $\delta \Delta H^{\neq}$int vs. $\delta \Delta G^{\neq}$for the $S_{N} 2$, acyl-transfer, $S_{N} A r$ and $A_{N}$ reactions of compounds $1-33$ with neutral amines $\mathbf{3 4 - 4 0}$ in various solvents; the identity of the numbers is the entry number in Table 1.

2S) can be explained by the existence of the zwitterionic tetrahedral intermediate $\mathbf{T}^{ \pm}$on the reaction pathway and a change in the RDS from formation of $\mathrm{T}^{ \pm}$ (step $k_{1}$ in Scheme 1) to its breakdown to products of $\mathbf{T}^{ \pm}$(step $k_{2}$ in Scheme 1), as the amine basicity decreases [44-53,59,60,64,77-109]. The low Brønsted slopes $\beta$ correspond to the formation of $\mathrm{T}^{ \pm}$(the step $k_{1}$ is the RDS) whereas the high ones determine the RDS with $k_{2}$ [44-53,59,60,64,77-109]. The middle value of the Brønsted slope $\beta$ is characteristic of the concerted mechanism in the aminolysis reactions $[40,41,44,45]$. In these cases, the Brønsted plot does not show a break [40-45,88]. In other words, a concerted mechanism has often been expected to give a straightline because a curved Brønsted plot can arise from a variable transition state [27].

At the same time, the sign and magnitude of the Hammett-like cross-interaction constants, $\rho_{\mathrm{RR}}$, where $R$ and $R^{\prime}$ are the substituents in the leaving and nonleaving groups and nucleophile, respectively, provide mechanistic criteria for the aminolysis reactions (Eq. 9) [40-53].

$\log \left(k_{\mathrm{RR}} / k_{\mathrm{HH}}\right)=\rho_{\mathrm{R}} \sigma_{\mathrm{R}}+\rho_{\mathrm{R}^{\prime}} \sigma_{\mathrm{R}^{\prime}}+\rho_{\mathrm{RR}} \sigma_{\mathrm{R}} \sigma_{\mathrm{R}^{\prime}}$

It was found that the cross-interaction constants $\rho_{\mathrm{RR}}$ ' are greater for the RDS with $k_{2}$ than the one with $k_{1}$ or $k_{\mathrm{c}}$ (entries $4-20,27-39,45-47,50-52,59-65$ in Table $2 \mathbf{S}$ of the supplementary data (Scheme 1) [10].

So, the analysis of the kinetic data on the basis of the Brønsted and Hammett-like equations gave an opportunity to determine the single RDS for the reactions of compounds $1-33$ with neutral amines 34 - 40 in solution (entries $1-65$ in Table 1). At the same time, the determination of the $k_{1}, k_{2}$ or $k_{\mathrm{c}}$ reaction rate constants as the single RDS makes it possible to estimate the role of the Eq. 8 for understanding the reaction mechanism.

It is obvious that the contribution of any step into the rate constant of the aminolysis with neutral amines in the BNRs can be determining. Therefore, the Eq. 8 has the more general character for the interpretation of the mechanistic features of these reactions. In particular, this dependence underlines the importance of the RDS operating on these BNRs. Furthermore, the dependence $\delta \Delta H^{\neq}$int $v s$. $\delta \Delta G^{\neq}$gives a possibility to elucidate the changes of the internal enthalpy $\delta \Delta H^{\neq}$int on the basis of the single reaction rate constants.

\section{Relationships between the Reaction Constants, $\delta \Delta H_{\text {int }}^{*}$ vs. $\rho$}

Considering Eqs. 7 and 8, a correlation between $\delta \Delta H^{\ddagger}$ int and $\rho$ for the reactions of entries $1-5,8,19$, 20, 23 - 26, 32 - 44, 50,. 53 - 58 in Table 1, for which the $k_{1}$ or $k_{2}$ step is presumed to be rate-determining, has been developed as shown by Eq. 10 (Figure 2). The Eq. 10 includes also the reactions of entries 6,7 , $9,10,21,22,30,31,45-49,59-65$ with the rate constants $k_{\mathrm{c}}$.

$\delta \Delta \mathrm{H}_{\text {int }}^{\neq}=(-0.07 \pm 0.24)-(6.16 \pm 0.10) \rho$ 


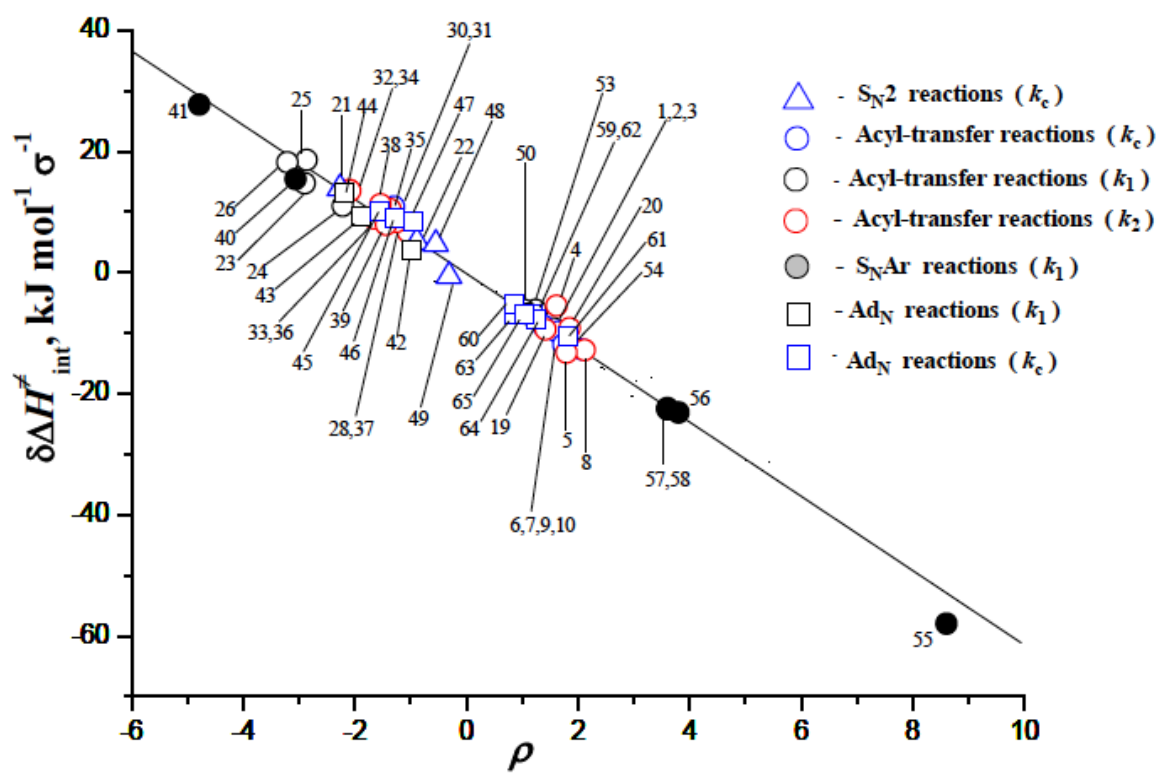

Figure 2: Plot of $\delta \Delta H^{\neq}$int vs. $\rho$ for the $S_{N} 2$, acyl-transfer, $S_{N} A r$ and $A_{N}$ reactions of compounds 1 - 33 with neutral amines $34-$ 40 in various solvents; the identity of the numbers is the entry number in Table 1.

The intercept in Eq. 10 is close to zero and the slope reflects a sensitivity of $\delta \Delta \mathrm{H}^{\neq}$int to a change of $\rho$ equaling approximately $2.303 \mathrm{RT}=-6.0$ at $T_{\text {exp }}=310-$ $317 \mathrm{~K}$. The single deviation from Eq. 10 for the reaction of entry 27 in Table 1 (Figure 2) is connected with an appreciable reduction of the $\delta \Delta \mathrm{H}^{\neq}$int reaction constant. Such reduction in the $\delta \Delta \mathrm{H}^{\neq}$int value is due to a larger positive change of an entropy component determined by the solvation influence of TS in protic solvent (see above) [64]. It is noteworthy that the calculated values of the $\delta \Delta \mathrm{H}^{\neq}$int reaction constants by Eq. 8 for entries 11 - 18, 28, 29, 51 and 52 in Table 1 coincide with the same values calculated by Eq. 10 .

Realization of Eq. 10 becomes possible, as magnitude of $\rho\left(\mathrm{k}_{1}\right), \rho\left(k_{2}\right)$ or $\rho\left(k_{\mathrm{c}}\right)$ for BNRs characterize charge development at the TS of the stepwise and concerted processes [23-26,67,110-113]. The $\delta \Delta H^{\neq}$int reaction constants characterize also the degree of developing charge in the TSs (rate constants $k_{1}, k_{2}$ or $k_{c}$ in Scheme 1). The large positive and negative values of $\delta \Delta \mathrm{H}^{\neq}$int indicate essential charge development in the TSs of $S_{N} A r$ reactions (entries 40,41 and 55 in Table 1) [31] and acyl-transfer ones (entries 25 and 26) (Figure 2). It is obvious that the electronic effects of substituents $R$ and $R^{\prime}$ in the leaving and nonleaving groups and nucleophiles are described by the single Hammett-like Eq. 10 for all BNRs with the rate constants $k_{1}, k_{2}$ or $k_{\mathrm{c}}$ when the rate constants $k_{1}$ or $k_{2}$ are rate-determining. The linearity over a wide range of the $\rho$ furthermore supports the assumption that there is no change in the mechanisms in this case. Therefore, the deviations from Eq. 10 can give a possibility to elucidate a reaction series in which a change of TS structure or reaction mechanism takes place.

\section{CONCLUSIONS}

The use of the parameters from the Brønsted and Hammett-like equations providing mechanistic criteria for the aminolysis in the BNRs has allowed us to determinate the single RDS in the mechanism of these reactions.

The reaction constants $\delta \Delta \mathrm{H}^{\neq}$int give rise to two linear dependences with the values of the reaction constants $\delta \Delta G^{\neq}$or the Hammett values of $\rho$ for the BNRs proceeding by a concerted mechanism and a stepwise mechanism through an intermediate with its formation or breakdown being the RDS. Furthermore, the different deviations from these dependences could have significant impact on the TS structures and mechanisms of the BNRs on the ground of their activation parameter variations.

The single linear dependence of $\delta \Delta H^{*}$ int $v s$. $\rho$ for all BNRs involving a change of substituent in the leaving and nonleaving groups and nucleophiles gives an opportunity to describe a very wide range of the Hammett $\rho$ values using the $\delta \Delta H^{\ddagger}$ int reaction constants.

\section{METHODS}

Eyring plots were generated by plotting $\log (\mathrm{k} / T)$ versus $1 / T$ using temperature-dependent rate data [40- 
53,64], and the enthalpies and entropies of activation, $\Delta H^{\neq}, \Delta S^{\neq}$, were determined for the BNRs of compound 1 - 33 with neutral amines 34 - 40 (Scheme 1). The activation parameters $\Delta \mathrm{H}^{\neq}, \Delta \mathrm{S}^{\neq}$, and $\Delta \mathrm{G}^{\neq}$obtained were used by Eq. 1 to establish the reaction constants $\delta \Delta \mathrm{H}^{\neq}, \delta \Delta \mathrm{S}^{\neq}$, and $\delta \Delta \mathrm{G}^{\neq}$( entries $4-20,28-39,45-$ 47, 50 - 52, $59-65$ in Tables 1 and $1 \mathrm{~S}$ in the supplementary data). These constants were then used in Eq. 2, 5 and 6 to determine the internal reaction constants $\delta \Delta H^{\neq}{ }_{\text {int }}$ when $\delta \Delta S_{\text {ext }}^{\neq} \approx 0$ and $\delta \Delta S^{\neq} \approx \delta \Delta S_{\text {ext }}^{\neq}$ (Table 1) [31-36]. The compensation Eq. 5 was tested at the $\geq 95 \%$ confidence level for all reaction series [31].

The quantitative data from the Brønsted and Hammett equations characterizing the aminolysis reactions of compounds $\mathbf{1}-\mathbf{3 3}$ are collected in Table 15 (in the supplementary data) to provide mechanistic criteria for these reactions $[40-53,64]$.

\section{SUPPLEMENTAL DATA}

The supplemental data can be downloaded from the journal website along with the article.

\section{REFERENCES}

[1] Kirk and Othmer Encyclopedia of Chemical Technology. 5th ed. New York: Wiley 2007; 18: p.524, 682 (see also references therein).

[2] Lawrence SA, Ed. Amines: Synthesis, Properties and Application. Cambridge: Cambridge University 2004.

[3] Smith MB, March J. March's Advanced Organic Chemistry 6th ed. New York: Wiley; 2007.

[4] Carey FA, Sundbery RJ. Advanced Organic Chemistry, Part A: Structure and Mechanisms. New York : Springer 2007.

[5] Williams A. Concerted Organic and Bio-organic Mechanisms. Boca Raton: CRC Press; 2000.

[6] Shaik SS, Schlegel HB, Wolfe S. Theoretical Aspects of Physical Organic Chemistry. New York: Wiley; 1992.

[7] Pross A. Theoretical and Physical Principles of Organic Reactivity. New York: Wiley; 1995.

[8] Anslyn EV, Dougherty DA. Modern Physical Organic Chemistry. Sausalito: University Science Books; 2006.

[9] Lee I. Characterization of transition state. Chem Soc Rev 1990; 19: 317-33

http://dx.doi.org/10.1039/cs9901900317

[10] Lee I, Sung DD. Theoretical and physical aspects. Curr Org Chem 2004; 8: 557-67.

[11] Castro EA Kinetics and mechanisms. Chem Rev 1999; 99: 3505-24.

[12] Bennet AJ, Brown RS. Physical organic chemistry. In: Sinnott $\mathrm{M}$, Ed. Comprehensive biological catalysis. New York: Academic Press 1998; vol. 1, p. 293 -326.

[13] Adler M, Adler S, Boche G. Tetrahedral intermediates. J Phys Org Chem 2005; 18: 193-209. http://dx.doi.org/10.1002/poc.807

[14] Terrier F. Modern nucleophilic aromatic substitution. Weinheim: Wiley-VCH Publishers; 2013. http://dx.doi.org/10.1002/9783527656141
[15] Buncel E, Dust JM, Terrier F. Rationalizing the regioselectivity. Chem Rev 1995; 95: 2261- 80. http://dx.doi.org/10.1021/cr00039a001

[16] Vlasov VM. Nucleophilic substitution. Russ Chem Rev 2003; 72: 681-703.

http://dx.doi.org/10.1070/RC2003v072n08ABEH000809

[17] Ji P, Atherton J, Page Ml. Organic reactivity. Org Biomol Chem 2012; 10: 5732-39. http://dx.doi.org/10.1039/c2ob25064k

[18] Bernasconi CF, Rappoport Z. Recent advances. Acc Chem Res 2009; 42: 993-1003. http://dx.doi.org/10.1021/ar900048q

[19] Bernasconi CF. Nucleophilic addition. Tetrahedron 1989; 45 4017-4090 http://dx.doi.org/10.1016/S0040-4020(01)81304-1

[20] Lee I. Secondary kinetic isotope effects. Chem Soc Rev 1995; 24: 223-29. http://dx.doi.org/10.1039/cs9952400223

[21] Hengge AC. Isotope effects. Acc Chem Res 2002; 35: 105112 http://dx.doi.org/10.1021/ar000143q

[22] Simmons EM, Hartwig JF. On the interpretation of deuterium kinetic isotope effects. Angew Chem Int Ed 2012; 51: 306672.

http://dx.doi.org/10.1002/anie.201107334

[23] Leffler JE, Grunwald E. Rates and equilibria of organic reaction. New York, London: Wiley; 1963.

[24] Hammett LP. Physical organic chemistry, reaction rates, equilibria and mechanisms. New York: McGraw Hill; 1970.

[25] Johnson CD. The Hammett Equation. Cambridge: Cambridge University Press; 1973.

[26] Williams A. Free energy relationships in organic and bioorganic chemistry. Cambridge: The Royal Society of Chemistry; 2003.

[27] Ammal SC, Mishima M, Yamataka $\mathrm{H}$. Linear free energy relationship. J Org Chem 2003; 68: 7772-78.

http://dx.doi.org/10.1021/j0034971

[28] Itoh S, Yamataka H. Dynamics effect. Chem Eur J 2011; 17 1230-37. http://dx.doi.org/10.1002/chem.201001926

[29] Itoh S, Yoshimura N, Sato M, Yamataka H. Computationa study. J Org Chem 2011; 76: 8294-99. http://dx.doi.org/10.1021/j0201485y

[30] Vlasov VM. Energetics of bimolecular nucleophilic reactions in solution. Russ Chem Rev 2006; 75: 765-96. http://dx.doi.org/10.1070/RC2006v075n09ABEH003614

[31] Vlasov VM. Towards mechanisms. J Phys Org Chem 2012; 25: 296-308. http://dx.doi.org/10.1002/poc.1912

[32] Vlasov VM. Effects of substituents. New J Chem 2009; 33 501-6. http://dx.doi.org/10.1039/b808115h

[33] Vlasov VM. Effects of substituents. J Phys Org Chem 2010; 23: 468-76.

[34] Vlasov VM. Substituent effects. New J Chem 2010; 34: 140816. http://dx.doi.org/10.1039/c0nj00058b

[35] Vlasov VM. Substituent effects. New J Chem 2010; 34: 2962 70 http://dx.doi.org/10.1039/c0nj00419g

[36] Vlasov, V. M. Substituent effects. Russ J Org Chem 2013 49: 391-97. http://dx.doi.org/10.1134/S1070428013030135

[37] Hepler LG. Effects of substituents. J Am Chem Soc 1963; 85 3089- 92. http://dx.doi.org/10.1021/ja00903a008 
[38] Hepler LG. Thermodynamic analysis. Can J Chem 1971; 49: 2803-07. http://dx.doi.org/10.1139/v71-466

[39] Ji P, Atherton J, Page Ml. Liquid ammonia. J Org Chem 2011; 76: 1425-35.

http://dx.doi.org/10.1021/j0102173k

[40] Oh HK, Yang JH, Sung DD, Lee I. Kinetics and mechanism. J Chem Soc Perkin Trans 2 2000; 101-05.

http://dx.doi.org/10.1039/a906639j

[41] Oh HK, Kim TS, Lee HW, Lee I. Kinetics and mechanism. J Chem Soc Perkin Trans 2 2002; 282-86.

http://dx.doi.org/10.1039/b108021k

[42] Oh HK, Kim IK, Lee HW, Lee I. Kinetics and mechanism. J Org Chem 2004; 69: 3806-10. http://dx.doi.org/10.1021/jo034370s

[43] Oh HK, Kim IK, Sung DD, Lee I. Kinetics and mechanism. Org Biomol Chem 2004; 2: 1213-16.

[44] Oh HK, Lee YH, Lee I. Kinetics and mechanism. Int J Chem Kinet 2000; 32: 131-5.

http://dx.doi.org/10.1002/(SICI)10974601(2000)32:3<131::AID-KIN2>3.0.CO;2-C

[45] Oh HK, Park JE, Sung DD, Lee I. Nucleophilic substitution. J Org Chem 2004; 69: 3150-53. http://dx.doi.org/10.1021/j0049845+

[46] Koh HJ, Kim SI, Lee BC, Lee I. Kinetics and mechanism. J Chem Soc Perkin Trans 2 1996; 1353-57. http://dx.doi.org/10.1039/p29960001353

[47] Oh HK, Yang JH, Cho IH, Lee HW, Lee I. Kinetics and mechanism. Int J Chem Kinet 2000; 32: 485-90. http://dx.doi.org/10.1002/1097-4601(2000)32:8<485::AIDKIN6>3.0.CO;2-X

[48] Koh HJ, Shin $\mathrm{CH}$, Lee HW, Lee I. Kinetics and mechanism. J Chem Soc Perkin Trans 2 1998; 1329-32. http://dx.doi.org/10.1039/a801540f

[49] Lee HW, Yun Y-S, Lee B-S, Koh HJ, Lee I. Kinetics and mechanism. J Chem Soc Perkin Trans 2 2000; 2302-05. http://dx.doi.org/10.1039/b002610g

[50] Koh HJ, Kim SK, Lee HW, Lee I. Structure - reactivity. J Chem Soc Perkin Trans 2 2001; 1753-57.

[51] Oh HK, Yang JH, Lee HW, Lee I. Kinetics and mechanism. Bull Korean Chem Soc 1999; 20: 1418-20.

[52] Oh HK, Kim SK, Lee I. Nucleophilic substitution. Bull Korean Chem Soc 1999; 20: 1017-20.

[53] Koh HJ, Han KL, Lee HW, Lee I. Kinetics and mechanism. J Org Chem 2000; 65: 4706-11. http://dx.doi.org/10.1021/j0000411y

[54] Mancini PME, Martinez RD, Vottero LR, Nudelman NS. Solvent effects. J Chem Soc Perkin Trans 2 1984; 1133- 38. http://dx.doi.org/10.1039/p29840001133

[55] Mancini PME, Martinez RD, Vottero LR, Nudelman NS. Solvent effects. J Chem Soc Perkin Trans 2 1987; 951-54.

[56] Crampton MR, Emokpae TA, Isanbor C. The effects of ring substituents. Eur J Org Chem 2007; 1378- 83. http://dx.doi.org/10.1002/ejoc.200600968

[57] Isanbor C, Emokpae TA Nucleophilic heteroaromatic substitution. Int. J. Chem. Kinet 2008; 40:125-35. http://dx.doi.org/10.1002/kin.20297

[58] Akinyele, E. T.; Onyido, I. J. Chem. Soc., Perkin Trans. 2 1988, 1859.

[59] Ji P, Atherton J, Page MI. The kinetics and mechanisms. J Org Chem 2011; 76: 3286-95. http://dx.doi.org/10.1021/jo200170z

[60] Um I-H, Im I-H, Kang J-S, Bursey SS, Dust JM. Mechanistic assessment. J Org Chem 2012; 77: 9738-46. http://dx.doi.org/10.1021/jo301862b
[61]

Um I-H, Min S-W, Dust JM. Choice of solvent. J Org Chem 2007; 72: 8797-8803.

http://dx.doi.org/10.1021/jo701549h

[62] Stegelmann C, Andreasen A, Campbell CT. Degree of rate control. J Am Chem Soc 2009; 131: 8077-82.

http://dx.doi.org/10.1021/ja9000097

[63] Kozuch S, Martin JML. The rate-determining step. ChemPhysChem 2011; 12: 1413-18. http://dx.doi.org/10.1002/cphc.201100137

[64] Castro EA, Santander CL. Nonlinear Brønsted-type plot. J Org Chem 1985; 50: 3595-600. http://dx.doi.org/10.1021/jo00219a029

[65] Hansch C, Leo A, Taft RW. A Survey of Hammett substituent. Chem Rev 1991; 91: 165-95. http://dx.doi.org/10.1021/cr00002a004

[66] Ruff F. Reaction constants. J Mol Des 2004; 3: 474-98.

[67] El Seoud OA, Ferreira M, Rodrigues WA, Ruasse M-F. Kinetics and mechanisms. J Phys Org Chem 2005; 18: 17382.

[68] Liu L, Guo QX. Isokinetic relationship.Chem Rev 2001; 101 : 673-95. http://dx.doi.org/10.1021/cr990416z

[69] Perez-Benito JF. Some tentative explanations. Monatsh Chem 2013; 144: 49-58. http://dx.doi.org/10.1007/s00706-012-0842-1

[70] Exner O. Entropy - enthalpy compensation. Chem Commun 2000; 1655-6.

http://dx.doi.org/10.1039/b002758h

[71] Linert W. Mechanistic and structural investigations. Chem Soc Rev 1994; 23: 429-38.

http://dx.doi.org/10.1039/cs9942300429

[72] Cornish-Bowden A. Ethalpy - entropy compensation J Biosci 2002; 27: 121-6.

http://dx.doi.org/10.1007/BF02703768

[73] Starikov EB, Nordén B. Enthalpy - entropy compensation. J Phys Chem B 2007; 111: 14431-35.

http://dx.doi.org/10.1021/jp075784i

[74] Starikov EB. Entropy is anthropomorphic. Monatsh Chem 2013; 144: 97-102.

http://dx.doi.org/10.1007/s00706-012-0837-y

[75] Starikov EB. Valid entropy-enthalpy compensation. J App Solution Chem Model 2013; 2: 240-45.

[76] Exner O. The enthalpy - entropy relationship. Prog Phys Org Chem 1973; 10: 411-82.

[77] Castro EA, Valdivia JL. Linear free-energy relationship. J Org Chem 1986; 51: 1668-72. http://dx.doi.org/10.1021/jo00360a007

[78] Castro EA, Vivanco M, Aguayo R, Santos JG. Kinetics and mechanism. J Org Chem 2004; 69: 5399-404. http://dx.doi.org/10.1021/jo049260f

[79] Castro EA, Aguayo R, Bessolo J, Santos JG. Kinetics and mechanism. J Org Chem 2005; 70: 3530-36. http://dx.doi.org/10.1021/j0050119w

[80] Castro EA, Aguayo R, Bessolo J, Santos JG. Kinetics and mechanism. J Phys Org Chem 2006;19: 555-61. http://dx.doi.org/10.1002/poc. 1055

[81] Castro EA, Acuňa $M$, Soto $C$, Trujillo $C$, Vàsquez $B$, Santos JG. Kinetics and mechanism. J Phys Org Chem 2008; 21 816-22. http://dx.doi.org/10.1002/poc.1399

[82] Castro EA, Bessolo J, Aguayo R, Santos JG. Kinetic investigation. J Org Chem 2003; 68: 8157-61. http://dx.doi.org/10.1021/jo0348120

[83] Castro EA, Aguayo R, Bessolo J, Santos JG. Kinetics and mechanism. J Org Chem 2005; 70: 7788-91. http://dx.doi.org/10.1021/j0051052f 
[84] Castro EA, Aliaga M, Campodónico PR, Cepeda M, Contreras R, Santos JG. Experimental and theoretical studies. J Org Chem 2009; 74: 9173-79. http://dx.doi.org/10.1021/j0902005y

[85] Castro EA, Millán D, Aguayo R, Campodónico PR, Santos JG. Reactions of aryl acetates. Int J Chem Kinet 2011; 43: 687-93.

http://dx.doi.org/10.1002/kin.20598

[86] Millán D, Santos JG, Castro EA. Kinetic study. J Phys Org Chem 2012; 25: 989-93.

http://dx.doi.org/10.1002/poc.2988

[87] Castro EA, Aliaga ME, Gazitúa M, Santos JG. The nucleofuge in the pyridinolysis. J Phys Org Chem 2012; 25: 994-97.

http://dx.doi.org/10.1002/poc.2989

[88] Castro EA, Ramos M, Santos JG. Concerted pyridinolysis. J Org Chem 2009; 74: 6374-77. http://dx.doi.org/10.1021/j0901137f

[89] Um I-H, Min J-S, Ahn J-A, Hahn H-J. Effect of acyl substituents. J Org Chem 2000; 65: 5659-63. http://dx.doi.org/10.1021/jo000482x

[90] Um I-H, Kim K-H, Park H-R, Fujio M, Tsuno Y. Effects of amine nature. J Org Chem 2004; 69: 3937-42. http://dx.doi.org/10.1021/jo049694a

[91] Um I-H, Lee J-Y, Lee HW, Nagano Y, Fujio M, Tsuno Y. Effect of o-methyl group. J Org Chem 2005; 70: 4980-87. http://dx.doi.org/10.1021/jo050172k

[92] Um I-H, Lee J-Y, Ko S-H, Bae S-K. Aminolysis of $\mathrm{Y}-$ substituted phenyl X-substituted benzoates. J Org Chem 2006; 71: 5800-03. http://dx.doi.org/10.1021/j00606958

[93] Um I-H, Jeon S-E, Seok J-A. Aminolysis of 2,4dinitrophenyl. Chem Eur J 2006; 12: 1237-43. http://dx.doi.org/10.1002/chem.200500647

[94] Um I-H, Lee J-Y, Fujio M, Tsuno Y. Structure - reactivity correlations. Org Biomol Chem 2006; 4: 2979-85. http://dx.doi.org/10.1039/b607194e

[95] Um I-H, Bae AR. Electronic nature. J Org Chem 2012; 77 : 5781-87.

http://dx.doi.org/10.1021/j0300961y

[96] Um I-H, Baek M-H, Han $\mathrm{H}-\mathrm{J}$. Effect of amine nature. Bull Korean Chem Soc 2003; 24: 1245-50.

[97] Um I-H, Hwang S-J, Baek M-H, Park EJ. Modification of both the electrophilic center. J Org Chem 2006; 71: 9191-97. http://dx.doi.org/10.1021/j0061682x

[98] Yew KH, Koh HY, Lee HW, Lee I. Nucleophilic substitution. J Chem Soc Perkin Trans 2 1995; 2263-68.

http://dx.doi.org/10.1039/p29950002263
[99] Koh HJ, Lee J-W, Lee HW, Lee I. Kinetics and mechanism. Can J Chem 1998; 76: 710-16.

http://dx.doi.org/10.1139/v98-038

[100] Oh HK, Kim SK, Cho IH, Lee HW, Lee I. Kinetics and mechanism. J Chem Soc Perkin Trans 2 2000; 2306-10. http://dx.doi.org/10.1039/b002842h

[101] Oh HK, Ha JS, Sung DD, Lee I. Aminolysis of aryl chlorothionoformates. J Org Chem 2004; 69: 8219-23. http://dx.doi.org/10.1021/jo0487247

[102] Oh HK, Park JE, Sung DD, Lee I. Kinetics and mechanism.. J Org Chem 2004; 69: 9285-88. http://dx.doi.org/10.1021/jo0484676

[103] Oh HK, Oh JY, Sung DD, Lee I. Aminolysis of aryl N-ethyl thionocarbamates. J Org Chem 2005; 70: 5624-29. http://dx.doi.org/10.1021/j0050606b

[104] Oh HK, Jin YC, Sung DD, Lee I. Kinetics and mechanism. Org Biomol Chem 2005; 3:1240-44. http://dx.doi.org/10.1039/b500251f

[105] Lee I, Lee HW, Yu Y-K. Kinetics and mechanism. Bull Korean Chem Soc 2003; 24: 993-98.

[106] Koh HJ, Han KI, Lee HW, Lee I. Kinetics and mechanism. J Org Chem 1998; 63: 9834- 39. http://dx.doi.org/10.1021/j09814905

[107] Koh HJ, Han KL, Lee I. Nucleophilic substitution. J Org Chem 1999; 64: 4783-89. http://dx.doi.org/10.1021/jo990115p

[108] Oh HK, Ku MH, Lee HW, Lee I. Kinetics and mechanism. J Org Chem 2002; 67: 8995-98. http://dx.doi.org/10.1021/jo0264269

[109] Koh HJ, Kang S-J, Kim CJ, Lee HW, Lee I. Kinetics and mechanism. Bull Korean Chem Soc 2003; 24: 925-30. http://dx.doi.org/10.5012/bkcs.2003.24.7.925

[110] Bernasconi CF, Michoff MEZ, de Rossi RH, Granados AM. Kinetics of the Reactions. J Org Chem 2007; 72: 1285-93. http://dx.doi.org/10.1021/jo062138r

[111] Bernasconi CF, Pérez-Lorenzo M, Codding SJ. Reactions of [aryloxy(phenyl)carbene]pentacarbonylchromium(o) complexes. J Org Chem 2007; 72: 9456-63. http://dx.doi.org/10.1021/jo701422z

[112] Kondo $Y$, Urade M, Yamanishi $Y$, Chen X. Relative reactivity J Chem Soc Perkin Trans 2 2002; 1449-54. http://dx.doi.org/10.1039/b203032m

[113] Edwards DR, Montoya - Peleaz P, Crudden CM. Experimental investigation. Org Lett 2007; 9: 5481-84. http://dx.doi.org/10.1021/ol702300d 